\title{
Phase ordering of the $O(2)$ model in the post-gaussian approximation
}

\author{
Robert A. Wickham and Gene F. Mazenko \\ The James Franck Institute and the Department of Physics \\ The University of Chicago \\ Chicago, Illinois 60637
}

(November 6, 2018)

\begin{abstract}
The gaussian closure approximation previously used to study the growth kinetics of the non-conserved $O(n)$ model is shown to be the zeroth-order approximation in a well-defined sequence of approximations composing a more elaborate theory. This paper studies the effects of including the next nontrivial correction in this sequence for the case $n=2$. The scaling forms for the order-parameter and order-parameter squared correlation functions are determined for the physically interesting cases of the $O(2)$ model in two and three spatial dimensions. The post-gaussian versions of these quantities show improved agreement with simulations. Post-gaussian formulae for the defect density and the defect-defect correlation function $\tilde{g}(x)$ are derived. As in the previous gaussian theory, the addition of fluctuations allows one to eliminate the unphysical divergence in $\tilde{g}(x)$ at short scaled-distances. The non-trivial exponent $\lambda$, governing the decay of order-parameter auto-correlations, is computed in this approximation both with and without fluctuations.
\end{abstract}

PACS numbers: 05.70.Ln, 64.60.Cn, 64.60.My, 64.75.+g 


\section{INTRODUCTION}

In the gaussian closure approximation for phase-ordering kinetics the order-parameter field is expressed in terms of an auxiliary field which is assumed to obey gaussian statistics [1.:2]. Recently, one of the authors extended this approach to treat more general statistics and applied the method successfully to the cases of conserved and non-conserved scalar fields [3, [4]. This post-gaussian approach successfully eliminated several shortcomings of the gaussian theory. The present paper has two primary goals. The first goal is to generalize the post-gaussian theory to treat the non-conserved $O(n)$ symmetric model with continuous symmetry $(n>1)$. Systems with continuous symmetry have many physical realizations, including ferromagnets, superfluids, and liquid crystals [5]. We will focus on the case $n=2$, where the defect-defect correlation function, $\tilde{g}(x)$, has an unphysical divergence at short scaled-distances, $x$, in the gaussian theory. While the post-gaussian theory weakens this divergence, it is not eliminated. The second goal of this paper is then to show that the inclusion of fluctuations counter-balance and thereby eliminate the divergence in $\tilde{g}(x)$ in the post-gaussian case.

In a phase-ordering scenario, the dynamical evolution of the order parameter $\vec{\psi}(1)=$ $\vec{\psi}\left(\mathbf{r}_{1}, t_{1}\right)$ is not typically governed by a gaussian probability distribution, and analytical progress up to now has relied on relating $\vec{\psi}(1)$ to an auxiliary field $\vec{m}(1)$, assumed to be gaussian. The gaussian approximation has been very successful in treating the scaling inherent in the late-time behaviour of a host of growth kinetics problems [6]. The gaussian theory predicts the now well-established result that for late times following a quench from the disordered to the ordered phase the dynamics obey scaling and the system can be described in terms of a single growing length

$$
L(t) \sim t^{\phi}
$$

which is characteristic of the spacing between defects at time $t$ after the quench. $\phi$ is a growth exponent. In this regime the scaling form $\mathcal{F}$ for the equal-time $\left(t_{1}=t_{2}=t\right)$ order-parameter correlation function 


$$
\begin{aligned}
C_{\psi}(12) & \equiv\langle\vec{\psi}(1) \cdot \vec{\psi}(2)\rangle \\
& =\psi_{0}^{2} \mathcal{F}(x)
\end{aligned}
$$

can be accurately calculated within the theory. Here $\psi_{0}$ is the magnitude $\psi=|\vec{\psi}|$ of the order-parameter in the ordered phase. The scaled length $x$ is defined as $x=r / L(t)$ with $r \equiv|\mathbf{r}| \equiv\left|\mathbf{r}_{2}-\mathbf{r}_{1}\right| . \quad$ For the non-conserved models considered here the growth exponent is $\phi=1 / 2$, which is predicted by the theory and well established by experiments and simulations [7]. The gaussian theory also makes quantitatively accurate predictions [2,8,9] for the exponent $\lambda$ governing the decay of the order-parameter auto-correlations, and defined by

$$
C_{\psi}\left(\mathbf{0}, t, t^{\prime}\right) \sim \frac{1}{L^{\lambda}(t)} \text { for } t \gg t^{\prime} .
$$

Finally, in addition to these accomplishments, the gaussian approximation is relatively easy to implement, and has straightforward generalizations to more complex systems.

Despite these achievements there remain a few unresolved issues. The approximate nature of gaussian statistics was highlighted in the work of Blundell, Bray, and Sattler (BBS) [10,11] where they computed, within the gaussian closure approximation, the twopoint correlation function for the square of the order-parameter field:

$$
C_{\psi^{2}}(12)=\frac{\left\langle\left[\psi_{0}^{2}-\psi^{2}(1)\right]\left[\psi_{0}^{2}-\psi^{2}(2)\right]\right\rangle}{\left\langle\psi_{0}^{2}-\psi^{2}(1)\right\rangle\left\langle\psi_{0}^{2}-\psi^{2}(2)\right\rangle}-1 .
$$

It is usual in comparing the theoretical scaling function $\mathcal{F}(x)$ with the data (both of which are relatively featureless) to rescale the length $x$ to give the best fit. This rescaling reflects the uncertainty in the relation of the theoretical pre-factor of the power law growth (1.1) to the pre-factor determined in experiment and simulation [12]. By plotting $1 /\left(C_{\psi^{2}}+1\right)$ against $1-\mathcal{F}$, hereafter referred to as a BBS plot, BBS were able to eliminate any adjustable fitting parameter and show that there were qualitative differences between the simulation results [11] and the predictions of existing gaussian theories (see Fig. [3). These discrepancies indicated a need to go beyond the gaussian approximation. Another motivation for going 
beyond the gaussian approximation is the ad hoc nature of the approximation itself and the desire to encompass it within a more general and systematic framework [13].

A second problem with the gaussian closure approximation occurs when one examines defect correlations. In this paper we focus on point defects $(n=d)$ whose density is defined as

$$
\rho(1)=\sum_{\alpha} q_{\alpha} \delta\left(\mathbf{r}_{1}-\mathbf{x}_{\alpha}\left(t_{1}\right)\right)
$$

where $\mathbf{x}_{\alpha}\left(t_{1}\right)$ is the position at time $t_{1}$ of the $\alpha$ th point defect, which has a topological charge $q_{\alpha}$. Defect-defect correlations

$$
\mathcal{G}(12) \equiv\langle\rho(1) \rho(2)\rangle
$$

at equal-times can be shown [14] to decompose into two parts

$$
\mathcal{G}(\mathbf{r}, t)=n_{0}(t) \delta(\mathbf{r})+g_{d d}(\mathbf{r}, t)
$$

The quantity $n_{0}(t)$ represents defect self-correlations and is just the total unsigned number density of defects at time $t$. We will be primarily concerned here with the defect-defect correlation function $g_{d d}(\mathbf{r}, t)$ which measures the correlations between different defects. The conservation law

$$
\int d^{n} r \mathcal{G}(\mathbf{r}, t)=0
$$

relates $n_{0}(t)$ and $g_{d d}(\mathbf{r}, t)$ through

$$
n_{0}(t)=-\int d^{n} r g_{d d}(\mathbf{r}, t)
$$

In the scaling regime it can be shown [14 that $n_{0}(t) \sim L^{-n}(t)$ and that $g_{d d}(\mathbf{r}, t)$ has the form

$$
g_{d d}(\mathbf{r}, t)=\frac{1}{L^{2 n}(t)} \tilde{g}(x)
$$

where $\tilde{g}(x)$ is a universal scaling function. While the form for $\tilde{g}(x)$ obtained from the gaussian theory for the two-dimensional $O(2)$ model is in good agreement with simulations 
[15] and experiments [16] at large scaled-distances, there are qualitative differences in the short scaled-distance behaviour. The gaussian theory [14] predicts a divergence in $\tilde{g}(x)$ at small $x$ while experiments and simulations have $\tilde{g}(x)$ approaching zero at the origin (see Fig. (1).

This paper addresses these issues for the non-conserved $O(n)$ model by using a welldefined sequence of approximations for the probability distribution of the auxiliary field $\vec{m}$, which reduces to a gaussian distribution at lowest order. The theory presented here treats in detail the next non-trivial term in the sequence. This post-gaussian approach has been successfully applied to both the cases of a non-conserved [3] and a conserved [4] scalar order parameter. While, as expected, the non-conserved scalar theory predicts little change in the form for $\mathcal{F}$, the BBS plot shows a marked improvement over the gaussian theory when compared with simulations. This encourages one extend the post-gaussian theory to the $O(n)$ case. The key result of this paper is that $\mathcal{F}, C_{\psi^{2}}, n_{0}(t), \tilde{g}(x)$ and $\lambda$ can all be extracted using non-gaussian statistics. This is non-trivial since these quantities have universal forms in the scaling regime. This is connected to the fact that the probability distribution governing the auxiliary field in the gaussian case has a fixed-point form determined by the solution of an eigenvalue problem. In the post-gaussian case the determination of the fixed-point form for the probability distribution requires the solution of a double eigenvalue problem. This paper focuses on the $O(2)$ model in two and three spatial dimensions where experimental and simulation results are readily available. For the $O(2)$ model in the post-gaussian approximation we find that the form for $\mathcal{F}$ changes little from the gaussian results and, as in the scalar case, the agreement of the BBS plot with simulations is improved. This improvement includes a dependence on dimensionality seen in the simulations, but not exhibited by the gaussian theory. There are some problems related to the negativity of $C_{\psi^{2}}$ at small $x$ but these are seen to be a consequence of the manner in which we defined the sequence of approximations. The exponent $\lambda$ is now in poorer agreement with the simulation data. The divergence in $\tilde{g}(x)$ is found to be weaker in the post-gaussian theory than in the gaussian theory, but it is not completely eliminated. 
We recently showed that this divergence can be eliminated in the gaussian case [17] if one includes the effects of fluctuations about the ordering field. The origin of the divergence is the appearance of non-analytic terms in the small- $x$ expansion of the auxiliary field correlation function $f$. One removes the divergence by eliminating the non-analytic terms in $f$ through a careful treatment of the fluctuations. This development is theoretically pleasing since one expects the auxiliary field correlation function to be well-behaved. The question remains whether this post-gaussian scheme can be smoothly generalized to include these fluctuations. We answer this question in the affirmative and see that the post-gaussian theory with fluctuations is a rather natural generalization of the gaussian theory. Again, the divergence in $\tilde{g}(x)$ is removed and the magnitude of $\tilde{g}(0)$ is reduced, bringing the postgaussian theory into better agreement with the simulation results [15] than the gaussian theory (see Fig. 四).

The first part of this paper is mainly devoted to developing the results for the postgaussian theory. Later, after we present the post-gaussian results for $\tilde{g}(x)$, we will discuss the role of fluctuations in detail. Section II reviews the gaussian $O(n)$ model and the separation of the equation of motion into an equation for the ordering field and an equation for the fluctuations. The main results of this paper are contained in Section III which, after introducing the post-gaussian probability distribution and general formulae for calculating post-gaussian averages, derives post-gaussian expressions for $\mathcal{F}, C_{\psi^{2}}$ and the equations of motion. Defect correlations are discussed, leading into Section IV which considers the inclusion of fluctuations in the post-gaussian theory. The results of numerical analysis of the new, post-gaussian eigenvalue problem are presented in Section V. We conclude with a summary and discussion.

\section{MODEL}




\section{A. Preliminaries}

We consider the $O(n)$ model, which describes the dynamics of a non-conserved, $n$ component order-parameter field $\vec{\psi}(1)=\left(\psi_{1}(1), \cdots, \psi_{n}(1)\right)$. To begin we will work with general $n$; however, later we will focus on the interesting case $n=2$. As in previous work in this area [2], the dynamics are modeled using a time-dependent Ginzburg-Landau equation

$$
\frac{\partial \vec{\psi}}{\partial t}=-\Gamma \frac{\delta F[\vec{\psi}]}{\delta \vec{\psi}} .
$$

We assume that the quench is to zero temperature where the usual noise term on the righthand side is zero $[18]$. $\Gamma$ is a kinetic coefficient and $F[\vec{\psi}]$ is the free-energy, assumed to be of the form

$$
F[\vec{\psi}]=\int d^{d} r\left(\frac{c}{2}|\nabla \vec{\psi}|^{2}+V[\psi]\right)
$$

where the potential $V[\psi]$ is chosen to have $O(n)$ symmetry and a degenerate ground state with $|\vec{\psi}|=\psi_{0}$ 19. With a suitable redefinition of the time and space scales the coefficients $\Gamma$ and $c$ can be set to one and (2.1) can be written as

$$
\frac{\partial \vec{\psi}}{\partial t}=\nabla^{2} \vec{\psi}-\frac{\partial V[\psi]}{\partial \vec{\psi}}
$$

It is believed that our final results are independent of the exact nature of the initial state, provided it is a disordered state.

The evolution induced by (2.3) causes $\vec{\psi}$ to order and assume a distribution that is far from gaussian. It is by now standard to introduce a mapping between the physical field $\vec{\psi}$ and an auxiliary field $\vec{m}$ with more tractable statistics. We can decompose $\vec{\psi}$ exactly as

$$
\vec{\psi}=\vec{\sigma}[\vec{m}]+\vec{u}
$$

The utility of this decomposition lies in our ability to create a consistent theory with the mapping $\vec{\sigma}$ chosen to reflect the defect structure in the problem, and the fluctuation correction $\vec{u}$ constructed to be small at late-times. The precise statistics satisfied by the fields $\vec{m}$ and $\vec{u}$ will be specified below. 
The mapping giving $\vec{\sigma}$ as a function of $\vec{m}$ is chosen to incorporate the dominant defect structure in the late-stage ordering kinetics. We assume that $\vec{\sigma}$ satisfies the Euler-Lagrange equation for the free-energy given by (2.2) with the spatial coordinate replaced by the auxiliary field:

$$
\nabla_{m}^{2} \vec{\sigma}[\vec{m}]=\frac{\partial V[\vec{\sigma}]}{\partial \vec{\sigma}} .
$$

The defects are then the non-uniform solutions of (2.5) which match on to the uniform solution at infinity. Since we expect only the lowest-energy defects, having unit topological charge, will survive to late-times the relevant solutions to (2.5) will be of the form

$$
\vec{\sigma}[\vec{m}]=A(m) \hat{m}
$$

where $m=|\vec{m}|$ and $\hat{m}=\vec{m} / m$. Thus the interpretation of $\vec{m}$ is that its magnitude represents the distance away from a defect core and its orientation indicates the direction to the defect core. We expect $m$, away from the defect cores, to grow as $L$ in the late-time scaling regime. Inserting (2.6) into (2.5) gives an equation for $A$,

$$
\nabla_{m}^{2} A-\frac{n-1}{m^{2}} A-V^{\prime}[A]=0,
$$

where the prime indicates a derivative with respect to $A$. The boundary conditions are $A(0)=0, A(\infty)=\psi_{0}$. An analysis of (2.7) for $n>1$ and large $m$ yields

$$
A(m)=\psi_{0}\left[1-\frac{\kappa}{m^{2}}+\cdots\right]
$$

where $\kappa=(n-1) / V^{\prime \prime}\left[\psi_{0}\right]>0$. The algebraic relaxation of the order-parameter to its ordered value is a distinct feature of the $O(n)$ model for $n>1$ which must be carefully treated in the evaluation of some averages. In the scalar case $(n=1) \psi$ relaxes exponentially to $\psi_{0}$ away from the defects.

\section{B. Separation of the equations of motion}

In [17] it was shown how one can quite generally separate the equation of motion (2.3) into an equation for the ordering field $\vec{\sigma}$ and an equation for the fluctuating field $\vec{u}$. One 
finds that $\vec{\sigma}$ satisfies the equation of motion

$$
\vec{B}=0
$$

with

$$
\vec{B} \equiv \partial_{t} \vec{\sigma}-\nabla^{2} \vec{\sigma}+\nabla_{m}^{2} \vec{\sigma}-\vec{\Theta}
$$

$\vec{\Theta}$ is chosen so that $\vec{u}$ is small in the scaling regime and thus represents a fluctuation. The equation of motion for $\vec{u}$ is

$$
\frac{\partial u_{i}}{\partial t}=\nabla^{2} u_{i}-W_{i j}[\vec{\sigma}] u_{j}-\Theta_{i}
$$

where a sum over the index $j$ is assumed and, to leading order in $1 / L$,

$$
W_{i j}[\vec{\sigma}]=q_{0}^{2} \hat{\sigma}_{i} \hat{\sigma}_{j}
$$

which is purely longitudinal. $q_{0}^{2}=V^{\prime \prime}\left[\psi_{0}\right]>0$.

If we set $\vec{\Theta}$ equal to zero in (2.10) we obtain the equation used previously to determine the $\vec{\sigma}$ correlations [2]. This choice decouples $\vec{\sigma}$ and $\vec{u}$. The equation for $\vec{u}$ would then separate into a (massless [20]) diffusion equation for the transverse piece $\vec{u}_{T}$ and an equation for the longitudinal piece $u_{L}$ with a mass term $-q_{0}^{2} u_{L}$. However, as was seen in the gaussian theory [2, 14, the equation for $\vec{\sigma}$ would necessarily lead to non-analytic behaviour in the short scaled-distance expansion for the normalized auxiliary field correlation function, $f(x)$, and would ultimately lead to an unphysical divergence in $\tilde{g}(x)$ at small $x$. We must choose $\vec{\Theta}$ so that our auxiliary field (or fields, in the post-gaussian theory) is analytic for small $x$. The possible forms we can use for $\vec{\Theta}$ are discussed in detail in [17]. The key ideas are that

(i) $\vec{\Theta}$ is chosen to be a function of $\vec{m}$ only. This choice means that $\vec{\sigma}$ satisfies a closed equation, while $\vec{u}$ is slaved by $\vec{m}$. This highlights the fact that, since we are working at zero temperature, it is $\vec{\Theta}$ and not temperature which is driving the fluctuations [21].

(ii) $\vec{\Theta}$ must be odd under $\vec{m} \rightarrow-\vec{m}$. 
(iii) $\vec{\Theta}$ must scale as $\mathcal{O}\left(L^{-2}\right)$ in the scaling regime if it is to compensate for the terms in the equation of motion which lead to the non-analyticities in $f$. This will also allow us to treat $\vec{u}$ as a fluctuation since it will imply $\vec{u} \sim L^{-2}$.

It is sufficient for our purposes to consider

$$
\vec{\Theta}=\frac{\omega_{0}}{L^{2}(t)} \vec{\sigma}
$$

where $\omega_{0}$ is a constant to be determined. This form will allow us to construct $f(x)$ to be analytic through terms of $\mathcal{O}\left(x^{2}\right)$. It was shown in [17] how (2.13) is the first term is a series for $\vec{\Theta}$ which can be used to enforce analyticity at $\mathcal{O}\left(x^{4}\right)$ and beyond.

\section{The gaussian approximation}

To complete the definition of the model one must specify the form of the probability distribution for the auxiliary field $\vec{m}$. Forcing $\vec{\sigma}$ to satisfy the exact equation of motion (2.9) is tantamount to solving the problem exactly, and will determine a probability distribution for $\vec{m}$ which is complicated and extremely difficult for purposes of computation. Progress can be made if one imposes the weaker constraint

$$
\langle\vec{B}(1) \cdot \vec{\sigma}(2)\rangle_{0}=0
$$

This equation allows one to insure that $\vec{B}(1)$ is reasonably small at late-times but gives one the flexibility to choose a suitable probability distribution. The simplest choice is a gaussian probability distribution for $\vec{m}$ :

$$
P[\vec{m}]=P_{0}[\vec{m}] \equiv \mathcal{N} e^{-K_{0}[\vec{m}]}
$$

with $\mathcal{N}$ a normalization constant and

$$
K_{0}[\vec{m}]=\frac{1}{2} \int d 1 d 2 C_{0}^{-1}(12) \vec{m}(1) \cdot \vec{m}(2)
$$

$C_{0}(12)$, the correlation function for the auxiliary field, is explicitly defined through 


$$
\left\langle m_{i}(1) m_{j}(2)\right\rangle_{0}=\delta_{i j} C_{0}(12) .
$$

Here we have used $\langle\cdots\rangle_{0}$ to indicate an average using the gaussian distribution $P_{0}[\vec{m}]$. Later, when we consider post-gaussian statistics, we will use $\langle\cdots\rangle$ to denote an average using the post-gaussian distribution $P[\vec{m}]$. The system is assumed to be statistically isotropic and homogeneous so $C_{0}(12)$ is invariant under interchange of its spatial indices. For future reference we also define the one-point correlation function

$$
S_{0}(1)=C_{0}(11)
$$

and the normalized auxiliary field correlation function

$$
f(12)=\frac{C_{0}(12)}{\bar{S}_{0}(12)}
$$

with $\bar{S}_{0}(12)=\sqrt{S_{0}(1) S_{0}(2)}$. As discussed above it is expected that both $C_{0}$ and $S_{0}$ grow as $L^{2}$ at late times.

In the gaussian theory the relationship between the auxiliary function $f$ and the observable functions $C_{\psi}, C_{\psi^{2}}$ and $\tilde{g}$ can be derived without reference to the dynamics contained in (2.14). Using (2.4), (2.6) and (2.8) $C_{\psi}$ can be written to leading order in $1 / L$ as

$$
\begin{aligned}
C_{\psi}(12) & =\psi_{0}^{2}\langle\hat{m}(1) \cdot \hat{m}(2)\rangle_{0} \\
& =\psi_{0}^{2} \mathcal{F}(12),
\end{aligned}
$$

with

$$
\mathcal{F}=\frac{n f}{2 \pi} B^{2}\left[\frac{1}{2}, \frac{n+1}{2}\right] F\left[\frac{1}{2}, \frac{1}{2} ; \frac{n+2}{2} ; f^{2}\right]
$$

where $B$ is the beta function and $F$ is the hypergeometric function [2,22]. For later convenience, we define the short-forms

$$
F_{1} \equiv F\left[\frac{1}{2}, \frac{1}{2} ; \frac{n+2}{2} ; f^{2}\right]
$$

and denote 


$$
F_{1}^{\prime} \equiv \frac{d F_{1}}{d f}, F_{1}^{\prime \prime} \equiv \frac{d^{2} F_{1}}{d f^{2}}, \ldots
$$

The quantity $C_{\psi^{2}}$ (1.4), as obtained by BBS [10] for $n>1$, is given by

$$
C_{\psi^{2}}=F\left[1,1 ; \frac{n}{2} ; f^{2}\right]-1
$$

For later notational simplicity we shall write

$$
F_{2}=F\left[1,1 ; \frac{n}{2} ; f^{2}\right]
$$

and, as with $F_{1}$, use the prime notation for differentiation with respect to $f$. As pointed out in [11], one can directly test the assumption of gaussian statistics, independent of the spatial form of $f$, by plotting $1 /\left(C_{\psi^{2}}+1\right)$ against $1-\mathcal{F}$. This is done for the gaussian $O(2)$ model in Fig. 3 and the discrepancy between the gaussian theory and the simulation data is evident. Since this discrepancy is due to the choice of gaussian statistics and not due to the method used to determine $f$ it strongly suggests that an improved choice of probability distribution is needed.

Within the gaussian theory, $\tilde{g}(x)$ is given by 14

$$
\tilde{g}(x)=n !\left(\frac{h}{x}\right)^{n-1} \frac{\partial h}{\partial x}
$$

with

$$
h=-\frac{\gamma}{2 \pi} \frac{\partial f}{\partial x}
$$

and $\gamma=1 / \sqrt{1-f^{2}}$. The defect density is

$$
n_{0}(t)=\frac{n !}{2^{n} \pi^{n / 2} \Gamma(1+n / 2)}\left[\frac{S_{0}^{(2)}}{n S_{0}(t)}\right]^{n / 2}
$$

with

$$
S_{0}^{(2)}=\frac{1}{n}\left\langle[\vec{\nabla} \vec{m}]^{2}\right\rangle_{0}
$$

In the gaussian theory without fluctuations $S_{0}^{(2)}=1$. The expressions $(2.26)$ and $(2.28)$ satisfy the conservation law (1.9). 
The constraint (2.14) determines the time evolution of the two-point order-parameter correlations. We use (2.14) to determine $f$ and $\mathcal{F}$, which are related through (2.21). Knowledge of $f$ allows us then to determine $C_{\psi^{2}}$ and $\tilde{g}$. To simplify the discussion we restrict ourselves initially to the case $\vec{\Theta}=0$, considered in [2]. The case when $\vec{\Theta}$ has the form (2.13) was addressed in [17, and later we will discuss the inclusion of fluctuations in some detail. After some manipulation (2.14) becomes

$$
\partial_{t_{1}} \mathcal{F}(12)-\nabla_{1}^{2} \mathcal{F}(12)-\frac{1}{S_{0}(1)} f \partial_{f} \mathcal{F}(12)=0
$$

Here we have used the short-hand notation $\partial_{f} \mathcal{F}=\partial \mathcal{F} / \partial f$. For $t_{1} \gg t_{2}$ both $\mathcal{F}$ and $f$ are small. In this limit (2.30) becomes a linear equation for $\mathcal{F}$ and, with the definition

$$
L^{2}(t)=\frac{\pi S_{0}(t)}{2 \mu}=4 t
$$

for the scaling length $L$ [23], $\lambda$ can be determined as [2,8]:

$$
\lambda=d-\frac{\pi}{4 \mu}
$$

To examine the equal-time order-parameter correlations in the late-time scaling regime we set $t_{1}=t_{2}=t$ and write 2.30 ) in terms of the scaled distance $x$. To leading order in $1 / L$ we have [2]

$$
\vec{x} \cdot \nabla_{x} \mathcal{F}+\nabla_{x}^{2} \mathcal{F}+\frac{\pi}{2 \mu} f \partial_{f} \mathcal{F}=0
$$

This is a non-linear eigenvalue problem with eigenvalue $\mu$, entering via the definition (2.31) for the scaling length. $\mu$ is determined by numerically matching the analytically determined behaviour at small- $x$ onto the analytically determined behaviour at large- $x$. For large $x$ both $\mathcal{F}$ and $f$ are small and $(2.33)$ can be linearized. In this regime the physical solution to $(2.33)$ is

$$
\mathcal{F} \sim x^{d-2 \lambda} e^{-x^{2} / 2}
$$

These results are valid for arbitrary $n>1$. Our focus, however, is on the $O(2)$ model where there are known qualitative discrepancies with simulation and experimental data [14 16]. 
With this in mind, we now examine the small- $x$ behaviour of the scaling equation (2.33) for the case $n=2$. For small- $x$ (2.33) admits the following general expansion for $f$ :

$$
f=1+f_{2} x^{2}\left[1+\frac{K_{2}}{\ln x}\left(1+\mathcal{O}\left[\frac{1}{\ln x}\right]\right)\right]+f_{4} x^{4}\left[1+\frac{K_{4}}{\ln x}\left(1+\mathcal{O}\left[\frac{1}{\ln x}\right]\right)\right]+\mathcal{O}\left(x^{6}\right) .
$$

Non-analyticities in $f$ appear as a result of the non-zero $K_{2}$ and $K_{4}$ coefficients multiplying factors of $1 / \ln x$.

The coefficients of the expansion (2.35) can be determined by examining (2.33) orderby-order at small $x$. Balancing terms at $\mathcal{O}(\ln x)$ gives

$$
f_{2}=-\frac{\pi}{4 \mu d}
$$

At $\mathcal{O}(1)$ one has

$$
K_{2}=-\frac{1}{d}
$$

The non-zero $K_{2}$ coefficient is particularly important since it is responsible for the divergence of the defect-defect correlation function at small $x$, as one can see by examining (2.26) at small- $x$ where one has, for $n=d=2$,

$$
\tilde{g}(x)=\frac{f_{2} K_{2}}{2 \pi^{2}} \frac{1}{x^{2}(\ln x)^{2}}+\cdots .
$$

\section{THE POST-GAUSSIAN THEORY}

\section{A. Beyond the gaussian approximation}

Any scheme that claims to improve upon the gaussian approximation should include the following elements: it should be systematic, with the gaussian approximation being 'lowest order', it should be fairly easy computationally, at least in the early stages, and it should be converging to the results of experiment and simulation the farther one goes in the scheme. We now present an approach which generalizes the previous gaussian theory and satisfies the first two criteria completely, and the third criterion in all areas save one. 
Since any functional can be written as a sum over generalized Hermite functional polynomials we write the probability distribution for the auxiliary field in the form [3]

$$
P[\vec{m}]=P_{0}[\vec{m}] \sum_{J=0}^{\infty} \sum_{i_{1}, \cdots, i_{J}} a_{J}\left(i_{1}, \cdots, i_{J} ; 1 \cdots J\right) H_{J}\left(i_{1}, \cdots, i_{J} ; 1 \cdots J\right)
$$

where the indices $i_{1} \cdots i_{J}$ each range from 1 to $n$. Integration over repeated spatial and temporal variables is assumed. $P_{0}[\vec{m}]$ is the gaussian distribution. The $H_{J}$ are the Hermite functional polynomials

$$
H_{J}\left(i_{1}, \cdots, i_{J} ; 1 \cdots J\right)=(-1)^{J} e^{K_{0}[\vec{m}]} \frac{\delta^{J}}{\delta m_{i_{1}}(1) \cdots \delta m_{i_{J}}(J)} e^{-K_{0}[\vec{m}]}
$$

which form a complete orthogonal set, spanning a space containing the $O(n)$ symmetric functionals [3]. The functions $a_{J}$, along with $C_{0}$, are determined by the symmetry of the problem and the series of constraints

$$
\begin{aligned}
\langle\vec{B}(1) \cdot \vec{\sigma}(2)\rangle & =0 \\
\left\langle B_{i}(1) \sigma_{j}(2) \sigma_{k}(3) \sigma_{l}(4)\right\rangle & =0
\end{aligned}
$$

In the gaussian theory $(J=0)$ only the first constraint (3.3) was necessary to completely determine the dynamics of $C_{0}$. As one does computations at higher $J$ more of these constraints are necessary to determine all the $a_{J}$. At each $J$ one has a systematic approach to the problem and one can, in principle, calculate to any order in $J$. One expects that by enforcing more constraints on $\vec{B}$ one will be satisfying the exact equation of motion (2.9) more stringently. At the same time one is developing a more accurate expression for $P[\vec{m}]$ as more Hermite polynomials are included. In this sense then the theory is expected to improve as one calculates to higher $J$. Finally, the use of Hermite polynomials allows one to straightforwardly express post-gaussian averages in terms of easily computable gaussian averages.

We will work to order $J=2$, which we call the first post-gaussian approximation, so we will make the choices 


$$
\begin{aligned}
a_{0} & =1 \\
a_{1}(i ; 1) & =0 \\
a_{2}(i, j ; 12) & =\delta_{i j} A_{2}(12) \\
a_{J}\left(i_{1}, \cdots, i_{J} ; 1 \cdots J\right) & =0 \text { for } J>2 .
\end{aligned}
$$

Here $A_{2}(12)$ is a scalar function, symmetric in its arguments. The condition on $a_{0}$ insures that the theory reduces to the correct gaussian limit at lowest order and normalizes the probability distribution, provided $P_{0}[\vec{m}]$ is normalized. The condition on $a_{1}$ reflects the fact that there are no external fields and $P[\vec{m}]=P[-\vec{m}]$. The choice for $a_{2}$ follows from the $O(n)$ symmetry, and considerations of isotropy and homogeneity.

\section{B. General results for post-gaussian averages}

In order to calculate physical quantities like $C_{\psi}$ in the post-gaussian approximation we must be able to express one- and two-point averages like $\langle\phi(1)\rangle$ and $\langle\phi(1) \chi(2)\rangle$, where $\phi$ and $\chi$ are functions of $\vec{m}$, in terms of related gaussian averages. The calculation for the case with no spatial gradients in the average is presented below. The important case where there are spatial gradients in the average is slightly more involved, but the results for the $O(n)$ model are straightforward generalizations of the results for the scalar case, presented in [3]. The two-point average $\langle\phi(1) \chi(2)\rangle$ can be related to gaussian averages by using the definitions (3.1) and (3.2) and doing a few integrations by parts. The result is

$$
\langle\phi(1) \chi(2)\rangle=\sum_{J=0}^{\infty} \sum_{i_{1}, \cdots, i_{J}} a_{J}\left(i_{1}, \cdots, i_{J} ; \overline{1} \cdots \bar{J}\right)\left\langle\frac{\delta^{J} \phi(1) \chi(2)}{\delta m_{i_{1}}(\overline{1}) \cdots \delta m_{i_{J}}(\bar{J})}\right\rangle_{0} .
$$

The barred quantities are integrated over in this expression, but they will only contribute if they take the values 1 or 2 . In the first post-gaussian approximation (3.5-3.8) the two-point average (3.9) is

$$
\begin{aligned}
& \langle\phi(1) \chi(2)\rangle=\langle\phi(1) \chi(2)\rangle_{0}+A_{2}(11)\left\langle\nabla_{m}^{2} \phi(1) \chi(2)\right\rangle_{0} \\
& +A_{2}(22)\left\langle\phi(1) \nabla_{m}^{2} \chi(2)\right\rangle_{0}+2 A_{2}(12)\left\langle\nabla_{m} \phi(1) \cdot \nabla_{m} \chi(2)\right\rangle_{0},
\end{aligned}
$$


with no integrations over the spatio-temporal variables. If we now proceed under the assumption that $A_{2}(11)$ and $A_{2}(22)$ are non-zero we will only be forced later to set them to zero so that the long-distance behaviour of the theory is physical. For notational convenience we set these quantities to zero at the outset. Equation (3.10) can be further simplified by the introducing the operator

$$
\hat{G}(12)=1+2 A_{2}(12) \frac{\partial}{\partial C_{0}(12)}
$$

since, for a general gaussian average $\langle\phi(1) \chi(2)\rangle_{0}$, the following identity holds:

$$
\left\langle\nabla_{m} \phi(1) \cdot \nabla_{m} \chi(2)\right\rangle_{0}=\frac{\partial}{\partial C_{0}(12)}\langle\phi(1) \chi(2)\rangle_{0}
$$

The two-point post-gaussian average then can be compactly written

$$
\langle\phi(1) \chi(2)\rangle=\hat{G}(12)\langle\phi(1) \chi(2)\rangle_{0}
$$

The operator notation illuminates the close relation between gaussian and post-gaussian

averages in the first post-gaussian approximation. By setting $\chi(2)=1$ in (3.10) one obtains the formula

$$
\langle\phi(1)\rangle=\langle\phi(1)\rangle_{0}
$$

for the one-point average in the first post-gaussian approximation.

\section{C. $C_{\psi}$ and $C_{\psi^{2}}$ in the first post-gaussian approximation}

The post-gaussian analogue of (2.20) for the order-parameter correlation function $C_{\psi}$ can be straightforwardly calculated using (3.13). We write

$$
\begin{aligned}
C_{\psi}(12) & =\psi_{0}^{2} \hat{G}(12)\langle\hat{m}(1) \cdot \hat{m}(2)\rangle_{0} \\
& =\psi_{0}^{2} \mathcal{F}(12)
\end{aligned}
$$

where now 


$$
\mathcal{F}=\frac{n}{2 \pi} B^{2}\left[\frac{1}{2}, \frac{n+1}{2}\right]\left[f F_{1}+2 g\left(F_{1}+f F_{1}^{\prime}\right)\right]
$$

The post-gaussian quantity $g$ is defined as

$$
g(12) \equiv \frac{A_{2}(12)}{\bar{S}_{0}}
$$

in analogy with the definition for $f(2.19)$. Although $f$ retains many features from the gaussian theory and $g$ carries much information about the post-gaussian corrections, later we will find that $f$ and $g$ influence each other strongly, and must be determined together using the appropriate constraint equations.

Equation (3.13) can also be used to determine the post-gaussian $C_{\psi^{2}}$ from the gaussian $C_{\psi^{2}}$. We have

$$
C_{\psi^{2}}=F_{2}+2 g F_{2}^{\prime}-1 \text { for } n \geq 2 \text {. }
$$

Unlike the situation in the gaussian theory, we now need to know the specific forms for $f$ and $g$ in order to create a BBS plot. Knowledge of the adjustable parameter in the length scale is, however, still unnecessary. Since the forms for $f$ and $g$ are needed in the BBS plot, one hopes that the shape of the BBS curve will now depend on spatial dimensionality. We should note at this point that if we had maintained $A_{2}(11) \neq 0$ we would now be forced to set it equal to zero so that both $C_{\psi}$ and $C_{\psi^{2}}$ decay to zero at large distances, as is expected physically.

\section{Equations of motion}

To determine the unknown functions $f$ and $g$ we will use the constraints (3.3) and (3.4), enforced to keep $\vec{B}$ small. For now, we will work with $\vec{\Theta}=0$ to make the post-gaussian analysis more transparent.

From our experience with the scalar case [3, 4], we know that the constraint equation (3.3) evaluated at a single space-time point,

$$
\langle\vec{B}(1) \cdot \vec{\sigma}(1)\rangle=0
$$


contains information about the short-distance behaviour of the theory that allows us later to simplify the constraint equations for $f$ and $g$. Written in full, (3.19) becomes

$$
\frac{1}{2} \partial_{t_{1}}\left\langle\sigma^{2}(1)\right\rangle-\left\langle\vec{\sigma}(1) \cdot \nabla_{1}^{2} \vec{\sigma}(1)\right\rangle+\left\langle\vec{\sigma}(1) \cdot \nabla_{m}^{2} \vec{\sigma}(1)\right\rangle=0
$$

Evaluating (3.20) to leading order, we have

$$
d_{0}^{(2)}=1 \text { for } n \geq 2
$$

where

$$
\begin{aligned}
& d_{0}^{(2)}=S_{0}^{(2)}+2 A_{2}^{(2)} \\
& S_{0}^{(2)} \equiv \lim _{1 \rightarrow 2}-\nabla_{1}^{2} C_{0}(12) \\
& A_{2}^{(2)} \equiv \lim _{1 \rightarrow 2}-\nabla_{1}^{2} A_{2}(12) .
\end{aligned}
$$

The definition (3.23) for $S_{0}^{(2)}$ is equivalent to (2.29) if one considers $(2.29)$ to be an average using a gaussian probability distribution with the post-gaussian $C_{0}(12)$ as its correlation function. The new constant $A_{2}^{(2)}$ will turn out to be determined as part of an eigenvalue problem. Since $S_{0}^{(2)}$ is a manifestly positive quantity, (3.21) also provides an important upper bound on the eigenvalue $A_{2}^{(2)}$. We must have $A_{2}^{(2)}<1 / 2$ for $n \geq 2$.

We now examine the constraint (3.3) written out in full,

$$
\partial_{t_{1}}\langle\vec{\sigma}(1) \cdot \vec{\sigma}(2)\rangle-\nabla_{1}^{2}\langle\vec{\sigma}(1) \cdot \vec{\sigma}(2)\rangle+\left\langle\left[\nabla_{m}^{2} \vec{\sigma}(1)\right] \cdot \vec{\sigma}(2)\right\rangle=0
$$

which in the gaussian case is the sole equation needed to determine the dynamics of $C_{\psi}$. The evaluation of (3.25) involves a straightforward application of (3.13) to the appropriate gaussian averages. At late times the leading order result is

$$
\partial_{t_{1}} \mathcal{F}(12)-\nabla_{1}^{2} \mathcal{F}(12)-\frac{n}{2 \pi S_{0}(1)} B^{2}\left[\frac{1}{2}, \frac{n+1}{2}\right]\left[(f+2 g)\left(F_{1}+f F_{1}^{\prime}\right)+2 g f\left(2 F_{1}^{\prime}+f F_{1}^{\prime \prime}\right)\right]=0 .
$$

For $t_{1} \gg t_{2} \mathcal{F}, f$ and $g$ are small and (3.26) again becomes a linear equation for $\mathcal{F}$. The relation between $\lambda$ and $\mu$ is (2.32), unchanged from the gaussian theory, and the definition of the scaling length $L(2.31)$ is retained. 
As in the gaussian case we can write (3.26) as an equal-time scaling equation

$$
\vec{x} \cdot \nabla_{x} \mathcal{F}+\nabla_{x}^{2} \mathcal{F}+\frac{n}{4 \mu} B^{2}\left[\frac{1}{2}, \frac{n+1}{2}\right]\left[(f+2 g)\left(F_{1}+f F_{1}^{\prime}\right)+2 g f\left(2 F_{1}^{\prime}+f F_{1}^{\prime \prime}\right)\right]=0 .
$$

Although the functions $\mathcal{F}, f$ and $g$ are related through (3.16) and (3.27), the additional constraint equation (3.4) is needed to complete the theory and determine these functions separately. In (3.4) we have a choice of how to contract the indices $i j k l$ and 1234 . It is important to note that the function $g$ entering the first post-gaussian theory is a two-point quantity. Thus, unlike the usual case in perturbation theory, the first-order corrections to the gaussian theory will not require us to treat the difficult intricacies of four-point correlation functions. Therefore, in order to determine $g$ we only need enforce (3.4) contracted to a two-point function. A non-trivial constraint is obtained by contracting the indices $i j k l$ in pairs. There then remain two possible constraint equations:

$$
\begin{aligned}
& \langle\vec{B}(1) \cdot \vec{\sigma}(1) \vec{\sigma}(2) \cdot \vec{\sigma}(2)\rangle=0 \\
& \langle\vec{B}(1) \cdot \vec{\sigma}(2) \vec{\sigma}(1) \cdot \vec{\sigma}(2)\rangle=0 .
\end{aligned}
$$

Unless there exists some degeneracy one cannot, in the first post-gaussian approximation, satisfy relations $(3.28)$ and $(3.29)$ simultaneously. Analysis of $(\overline{3.29})$ shows that for $n=2$ it produces an equation in which the eigenvalue $A_{2}^{(2)}$ does not appear [24]. This is contrary to our expectation, based on previous work [3, [4, that we have to solve a double eigenvalue problem in the post-gaussian theory. It is therefore clear that we should satisfy (3.28).

Since we are enforcing (3.19) we may rewrite (3.28) as

$$
\langle\vec{B}(1) \cdot \vec{\sigma}(1) \Delta(2)\rangle=0
$$

where

$$
\Delta(2)=\sigma^{2}(2)-\psi_{0}^{2}
$$

This computationally convenient form allows the calculation to proceed in a way similar to that in [3]. Written in full, (3.30) becomes 


$$
\left\langle\left[\partial_{t_{1}} \vec{\sigma}(1)\right] \cdot \vec{\sigma}(1) \Delta(2)\right\rangle-\left\langle\left[\nabla_{1}^{2} \vec{\sigma}(1)\right] \cdot \vec{\sigma}(1) \Delta(2)\right\rangle+\left\langle\left[\nabla_{m}^{2} \vec{\sigma}(1)\right] \cdot \vec{\sigma}(1) \Delta(2)\right\rangle=0 .
$$

To evaluate the post-gaussian averages in (3.32) one must use the formulae given in Section III.B and generalize the results in [3] for post-gaussian averages containing spatial gradients of a scalar field $m$ to the case of a vector field $\vec{m}$. Two new gaussian averages specific to the $O(n)$ model must be calculated. After some algebra and rearrangement, (3.32) reduces at late-times to an equal-time scaling equation for $f$ and $g$. For $n=2$ we have, at leading order in $1 / L$,

$$
4 \pi A_{2}^{(2)} g f+4 \mu \nabla_{x} f \nabla_{x} g+\mu\left(\nabla_{x} f\right)^{2}\left(1+8 g f \gamma^{2}\right)=0
$$

For $n>2$ we have

$$
\begin{aligned}
& 4 \pi A_{2}^{(2)} g f^{2} F_{2}^{\prime} / \gamma^{2}+4 \mu \nabla_{x} f \nabla_{x} g\left[2 f^{2} F_{2}-(n-2)\left(F_{2}-1\right)\right]+ \\
& \mu\left(\nabla_{x} f\right)^{2}\left[2 f^{2} F_{2}-(n-2)\left(F_{2}-1\right)+2 g\left(4 \gamma^{2} f^{3} F_{2}+2 f^{2} F_{2}^{\prime}-\right.\right. \\
& \left.\left.(n-2)\left(F_{2}^{\prime}+2\left(\gamma^{2}-2\right)\left(F_{2}-1\right) / f\right)\right)\right]=0 .
\end{aligned}
$$

Note that for $n=2, F_{2}=\gamma^{2}$ and (3.34) reduces to (3.33).

These equations, together with (3.16) and (3.27) form a complete set of relations that will be used to determine the functions $\mathcal{F}, f$ and $g$. There are two unspecified constants in this set of equations - $\mu$ and $A_{2}^{(2)}$. We thus have a non-linear eigenvalue problem in which $\mu$, the eigenvalue familiar from the gaussian theory, and the new eigenvalue $A_{2}^{(2)}$ are determined by connecting the small- and large- $x$ behaviour of (3.27) and (3.34).

For large $x$ (3.27) reduces to a linear equation, as in the gaussian case, and once again leads to (2.34). The form for the exponent $d-2 \lambda$ in (2.34) appears to be robust. An examination of equation (3.34) at large- $x$ yields

$$
f=-4 g
$$

Up to now these results have been valid for $n>1$. Now, in examining the short scaleddistance properties, we will focus on the $n=2$ case where there are logarithmic corrections. The generalization of $(2.35)$ is 


$$
\begin{aligned}
& f(x)=1+f_{2} x^{2}\left[1+\frac{K_{2}}{\ln x}\left(1+\mathcal{O}\left[\frac{1}{\ln x}\right]\right)\right]+f_{4} x^{4}\left[1+\frac{K_{4}}{\ln x}\left(1+\mathcal{O}\left[\frac{1}{\ln x}\right]\right)\right]+\mathcal{O}\left(x^{6}\right) \\
& g(x)=g_{2} x^{2}\left[1+\frac{L_{2}}{\ln x}\left(1+\mathcal{O}\left[\frac{1}{\ln x}\right]\right)\right]+g_{4} x^{4}\left[1+\frac{L_{4}}{\ln x}\left(1+\mathcal{O}\left[\frac{1}{\ln x}\right]\right)\right]+\mathcal{O}\left(x^{6}\right)
\end{aligned}
$$

An examination of (3.33) yields, at $\mathcal{O}\left(x^{2}\right)$,

$$
\pi A_{2}^{(2)} g_{2}+\mu\left(f_{2}\right)^{2}=0
$$

At $\mathcal{O}\left(x^{2} / \ln x\right)$ one has

$$
L_{2}=2 K_{2}
$$

Examining (3.27) yields, at $\mathcal{O}(\ln x)$,

$$
f_{2}+2 g_{2}=-\frac{\pi}{4 \mu d},
$$

while at $\mathcal{O}(1)$, using (3.39) one has

$$
K_{2}=-\frac{1}{2}+\frac{\pi}{4 \mu d\left(f_{2}+4 g_{2}\right)}\left[\frac{2-d}{2 d}-\frac{g_{2}}{f_{2}}\right] .
$$

Equations (3.38) and (3.40) determine $f_{2}$ and $g_{2}$ separately in terms of the eigenvalues $\mu$ and $A_{2}^{(2)}$

$$
\begin{aligned}
& f_{2}=\frac{\pi}{4 \mu d}\left[A_{2}^{(2)} d-\sqrt{A_{2}^{(2)} d\left(2+A_{2}^{(2)} d\right)}\right] \\
& g_{2}=-\frac{\pi}{8 \mu d}\left[1+A_{2}^{(2)} d-\sqrt{A_{2}^{(2)} d\left(2+A_{2}^{(2)} d\right)}\right] .
\end{aligned}
$$

We have assumed that $A_{2}^{(2)}>0$ and taken the negative square root in (3.42) in order to render $f_{2}$ negative, as we expect $f \leq 1$ physically. Equations (3.39) and (3.41) determine the corrections to the leading-order behaviour. The small- $x$ expansion of the scaling form (3.18) for $C_{\psi^{2}}$ is

$$
C_{\psi^{2}}(x)=\frac{2 g_{2}-f_{2}}{2\left(f_{2}\right)^{2}} \frac{1}{x^{2}}+\frac{K_{2}}{2 f_{2}} \frac{1}{x^{2} \ln x}+\mathcal{O}\left[\frac{1}{(x \ln x)^{2}}\right] .
$$


Note that the condition for $C_{\psi^{2}}$ to be positive near the origin, the behaviour expected physically, is $2 g_{2}-f_{2}>0$ which is true for

$$
A_{2}^{(2)}>1 / 4 d
$$

We will find, however, that $A_{2}^{(2)}$ does not satisfy this lower bound, and the resulting negativity of $C_{\psi^{2}}$ has to be interpreted carefully.

\section{E. Defect correlations}

The starting point for evaluating defect-defect correlations is to note [14] that the density for point defects (1.5) can be rewritten in terms of the order-parameter field,

$$
\rho(1)=\delta[\vec{\psi}(1)] \operatorname{det}\left[\vec{\nabla}_{1} \vec{\psi}(1)\right]
$$

since the zeros of $\vec{\psi}$ are the locations of the defects. From (2.6) and (2.7) we see that $\vec{\psi} \sim \vec{m}$ near the defect cores so (3.46) can be expressed in terms of the auxiliary field

$$
\rho(1)=\delta[\vec{m}(1)] \operatorname{det}\left[\vec{\nabla}_{1} \vec{m}(1)\right]
$$

This form is convenient for evaluating the equal-time defect-defect correlations (1.6) which separate as indicated in (1.7) into a piece representing the defect self-correlations,

$$
n_{0}(t)=\left\langle\delta[\vec{m}(1)]\left|\operatorname{det}\left[\vec{\nabla}_{1} \vec{m}(1)\right]\right|\right\rangle,
$$

and a piece representing correlations between different defects

$$
g_{d d}(\mathbf{r}, t)=\left\langle\delta[\vec{m}(1)] \delta[\vec{m}(2)] \operatorname{det}\left[\vec{\nabla}_{1} \vec{m}(1)\right] \operatorname{det}\left[\vec{\nabla}_{2} \vec{m}(2)\right]\right\rangle .
$$

We sketch the calculation of $g_{d d}(\mathbf{r}, t)$ for the post-gaussian theory in the Appendix. We recover the scaling relation (1.10) with the post-gaussian scaling form

$$
\tilde{g}(x)=n !\left(\frac{h}{x}\right)^{n-1}\left(\frac{\partial h}{\partial x}+\frac{\partial \bar{h}}{\partial x}\right)+(n-1) n !\left(\frac{h}{x}\right)^{n-2} \frac{\bar{h}}{x} \frac{\partial h}{\partial x}
$$


where $h$ has the same definition it had in the gaussian theory (2.27) and $\bar{h}$, which contains the new post-gaussian terms, is given by

$$
\bar{h}=-\frac{\gamma}{\pi}\left(\frac{\partial g}{\partial x}+g f \gamma^{2} \frac{\partial f}{\partial x}\right)
$$

The defect density $n_{0}(t)$ can be calculated directly by evaluating the post-gaussian average in (3.48). The derivation is similar to that given for the gaussian theory in 14 and, as in the gaussian theory, the absolute value of the determinant appearing in (3.48) is a complication

that has to be carefully treated. Alternatively, we can use the conservation law (1.9) to compute $n_{0}(t)$, using the post-gaussian formulae (3.50) for $\tilde{g}(x)$. Both approaches lead to the same result:

$$
n_{0}(t)=\frac{n !}{2^{n} \pi^{n / 2} \Gamma(1+n / 2)}\left[\frac{S_{0}^{(2)}}{n S_{0}(t)}\right]^{n / 2}\left[1+n \frac{A_{2}^{(2)}}{S_{0}^{(2)}}\right] .
$$

Using the small- $x$ expansions (3.36) and (3.37) for $f$ and $g$ we calculate the post-gaussian modification to the behaviour of $\tilde{g}$ at small $x$ for $n=d=2$ :

$$
\tilde{g}(x)=\frac{\left(f_{2}+4 g_{2}\right) K_{2}}{2 \pi^{2}} \frac{1}{x^{2}(\ln x)^{2}}+\cdots
$$

Thus, as in the gaussian theory, leading order non-analyticities in the small- $x$ behaviour of the post-gaussian $f$ and $g$ are responsible for an unphysical divergence in $\tilde{g}(x)$ at small$x$. We will see that in the post-gaussian theory the divergence is weaker than it was in the gaussian theory; however, through the inclusion of fluctuations we can eliminate the divergence altogether.

\section{THE POST-GAUSSIAN THEORY INCLUDING FLUCTUATIONS}

\section{A. Analysis of the $\vec{\sigma}$ degrees of freedom}

We will now show how the inclusion of fluctuations about the ordering field $\vec{\sigma}$ can be used to eliminate the leading order non-analyticities in the small- $x$ behaviour of $f$ and $g$, thereby rendering $\tilde{g}(0)$ finite. This has been done successfully for the gaussian theory in 
[17]. Fluctuations influence the equation of motion for $\vec{\sigma}$ (2.9) through the non-zero $\vec{\Theta}$ field (2.13). The one-point equation (3.20), used to determine $d_{0}^{(2)}$ is modified by fluctuations to

$$
\frac{1}{2} \partial_{t_{1}}\left\langle\sigma^{2}(1)\right\rangle-\left\langle\vec{\sigma}(1) \cdot \nabla_{1}^{2} \vec{\sigma}(1)\right\rangle+\left\langle\vec{\sigma}(1) \cdot \nabla_{m}^{2} \vec{\sigma}(1)\right\rangle-\frac{\omega_{0}}{L^{2}\left(t_{1}\right)}\left\langle\sigma^{2}(1)\right\rangle=0 .
$$

The only difference between (4.1) and (3.20) is the last term, which is $\mathcal{O}\left(L^{-2}\right)$. For $n=2$ the leading order contribution to (4.1) is $\mathcal{O}\left(\ln L / L^{2}\right)$ so the fluctuation term does not modify the result $d_{0}^{(2)}=1$ for $n=2$ in the post-gaussian theory. A similar thing happens in the gaussian theory where the inclusion of fluctuations does not modify the relation $S_{0}^{(2)}=1$ for $n=2$ [17.

Fluctuations do, however, modify the two-point equation of motion (3.25) determining the order-parameter correlations and lead to a new formula for $\lambda$ :

$$
\lambda=d-\frac{\pi}{4 \mu}-\frac{\omega_{0}}{2} .
$$

At equal-times in the scaling regime (3.25), including fluctuations, becomes

$$
\vec{x} \cdot \nabla_{x} \mathcal{F}+\nabla_{x}^{2} \mathcal{F}+\omega_{0} \mathcal{F}+\frac{n}{4 \mu} B^{2}\left[\frac{1}{2}, \frac{n+1}{2}\right]\left[(f+2 g)\left(F_{1}+f F_{1}^{\prime}\right)+2 g f\left(2 F_{1}^{\prime}+f F_{1}^{\prime \prime}\right)\right]=0 .
$$

The final equation of constraint (3.32) is modified to

$$
\left\langle\left[\partial_{t_{1}} \vec{\sigma}(1)\right] \cdot \vec{\sigma}(1) \Delta(2)\right\rangle-\left\langle\left[\nabla_{1}^{2} \vec{\sigma}(1)\right] \cdot \vec{\sigma}(1) \Delta(2)\right\rangle-\frac{\omega_{0}}{L^{2}\left(t_{1}\right)}\left\langle\vec{\sigma}^{2}(1) \Delta(2)\right\rangle+\left\langle\left[\nabla_{m}^{2} \vec{\sigma}(1)\right] \cdot \vec{\sigma}(1) \Delta(2)\right\rangle=0,
$$

however a calculation for $n=2$ shows that at leading order in $1 / L$, this equation is unchanged from (3.33), even in the presence of fluctuations. Again, one has

$$
4 \pi A_{2}^{(2)} g f+4 \mu \nabla_{x} f \nabla_{x} g+\mu\left(\nabla_{x} f\right)^{2}\left(1+8 g f \gamma^{2}\right)=0 .
$$

The large- $x$ behaviour of the theory with fluctuations is essentially unchanged from the original post-gaussian theory. Equations (2.34) and (3.35) still hold except now $\lambda$ is given by (4.2). The expansions (3.36) and (3.37) are used to examine the small- $x$ behaviour of 
equations (4.3) and (4.5). Once again $f_{2}$ and $g_{2}$ are given by (3.42) and (3.43). The relation (3.39) still holds and from (4.3) one has

$$
\omega_{0}=g_{2}\left(2 d+\frac{\pi}{2 \mu f_{2}}\right)-\frac{\pi}{2 \mu d}+2 d K_{2}\left(f_{2}+4 g_{2}\right) .
$$

At this point we insist that $f$ and $g$ are analytic for small- $x$ at $\mathcal{O}\left(x^{2}\right)$ (i.e. $K_{2}=L_{2}=0$ ). This then fixes $\omega_{0}$ in terms of $\mu$ and $A_{2}^{(2)}$ :

$$
\omega_{0}=g_{2}\left(2 d+\frac{\pi}{2 \mu f_{2}}\right)-\frac{\pi}{2 \mu d} .
$$

The next order terms are $\mathcal{O}\left(x^{4}\right)$. From (4.3) and (4.5) we obtain two equations determining $f_{4}$ and $g_{4}$ :

$$
\begin{gathered}
\left(\pi A_{2}^{(2)}+4 \mu f_{2}\right) g_{4}+4 \mu\left(f_{2}-g_{2}\right) f_{4}=2 \mu g_{2}\left(f_{2}\right)^{2}-\pi A_{2}^{(2)} g_{2} f_{2} \\
f_{4}+2 g_{4}=\frac{3}{4}\left(f_{2}\right)^{2}+3 f_{2} g_{2}+\frac{\left(f_{2}+2 g_{2}\right)}{4(d+2)}\left[\frac{\pi}{4 \mu}-2-\omega_{0}\right] .
\end{gathered}
$$

Similarly, at the next order two relations can be derived for $K_{4}$ and $L_{4}$, which are more involved.

By using $\omega_{0}$ to eliminate the terms at $\mathcal{O}\left(x^{2} / \ln x\right)$ in $f$ and $g$ we have also managed to remove the divergence in $\tilde{g}(x)$ at the origin. For small- $x$ we now have

$$
\tilde{g}(x)=-\frac{1}{\pi^{2}}\left(3\left(f_{4}+2 g_{4}\right)-\frac{\left(f_{2}\right)^{2}}{2}-2 f_{2} g_{2}\right)+\mathcal{O}\left(\frac{1}{\ln x}\right) .
$$

\section{B. Analysis of fluctuation correlations}

Correlations in the fluctuation field $\vec{u}$ are completely determined within the theory. There are two types of equal-time averages that are of interest to us. The first describes crosscorrelations between the $\vec{\sigma}$ and $\vec{u}$ fields and is defined as

$$
C_{u 0}(12)=\left\langle\vec{u}\left(\mathbf{r}_{1}, t\right) \cdot \vec{\sigma}\left(\mathbf{r}_{2}, t\right)\right\rangle
$$

The second describes correlations of the fluctuation field with itself and is given by 


$$
\delta_{i j} C_{u u}(12)=\left\langle u_{i}\left(\mathbf{r}_{1}, t\right) u_{j}\left(\mathbf{r}_{2}, t\right)\right\rangle
$$

As we will see later these quantities are closely related in the scaling regime. In [17] it was shown how one can form equations of motion for both $C_{u 0}$ and $C_{u u}$ by using the equations of motion (2.9) and (2.11) for $\vec{\sigma}$ and $\vec{u}$. We can then use these equations to determine $C_{u 0}$ and $C_{u u}$ explicitly if we make the additional assumption that $\vec{u}$ is a gaussian field coupled to the post-gaussian field $\vec{m}$. To effect this change we replace the gaussian functional $e^{-K_{0}[\vec{m}]}$ in equations (3.1) and (3.2) by the gaussian functional $e^{-K_{0}[\vec{m}, \vec{u}]}$ which is quadratic in both $\vec{m}$ and $\vec{u}$. Post-gaussian averages over $\vec{m}$ are evaluated as before, while repeated use of the following identity

$$
\left\langle u_{i}(1) \mathcal{A}[\vec{m}, \vec{u}]\right\rangle=\int d 3 C_{u m}(13)\left\langle\frac{\delta}{\delta m_{i}(3)} \mathcal{A}[\vec{m}, \vec{u}]\right\rangle+\int d 3 C_{u u}(13)\left\langle\frac{\delta}{\delta u_{i}(3)} \mathcal{A}[\vec{m}, \vec{u}]\right\rangle,
$$

with

$$
\delta_{i j} C_{u m}(12)=\left\langle u_{i}(1) m_{j}(2)\right\rangle
$$

allows averages over $\vec{u}$ to be expressed in terms $C_{u u}, C_{u m}$ and post-gaussian averages over $\vec{m}$. We then evaluate the averages over $\vec{m}$, which can be expressed in terms of $f$ and $g$ obtained previously. Finally, we examine the equations of motion for the fluctuations in the late-time scaling regime and extract the scaling functions. The analysis closely follows that given in [17] so here we report only the final results for the post-gaussian theory.

In [17] it was shown that, as a consequence of the definition (2.13) for $\vec{\Theta}$, we must have $u \sim L^{-2}$ to leading order. We therefore write the scaling relations

$$
C_{u 0}(12)=\frac{\psi_{0}^{2}}{L^{2}} F_{u}(x)
$$

and

$$
C_{u u}(12)=\frac{\psi_{0}^{2}}{L^{4}} F_{u u}(x)
$$

The equations of motion developed in [17] can be generalized to the post-gaussian case and produce the following relations between $F_{u}$ and $F_{u u}$ : 


$$
\begin{aligned}
& F_{u}(0)\left[\frac{1}{f}\left(1-\sqrt{1-f^{2}}\right)+2 g \frac{1-\sqrt{1-f^{2}}}{f^{2} \sqrt{1-f^{2}}}\right] \\
& +F_{u}(x)\left[\frac{\sqrt{1-f^{2}}}{1+\sqrt{1-f^{2}}}-\frac{2 g f}{\sqrt{1-f^{2}}\left(1+\sqrt{1-f^{2}}\right)^{2}}\right]=-\frac{\omega_{0}}{q_{0}^{2}} \mathcal{F}(x)
\end{aligned}
$$

and

$$
F_{u u}(x)=-\frac{1}{q_{0}^{2}}\left[\omega_{0}+\frac{2 q_{0}^{2}}{\pi} F_{u}(0)\right] F_{u}(x) .
$$

For $g=0$ (4.17) simplifies to the gaussian form found in [17]. The quantity $F_{u}(0)$ enters into these equations and an analysis of (4.17) at $x=0$ gives

$$
F_{u}(0)=-\frac{\omega_{0}}{q_{0}^{2}} .
$$

We then find that

$$
\mathcal{F}_{u u}(0)=\frac{\omega_{0}^{2}}{q_{0}^{4}}\left[1-\frac{2}{\pi}\right]>0,
$$

which is a necessary condition for stability and is expected from the definitions (4.12) and (4.16). Equations (4.17) and (4.18) explicitly show how correlations in the $\vec{u}$ field are slaved to those of the order parameter. We have demonstrated here that the theory is consistent and that the fluctuations remain of $\mathcal{O}\left(L^{-2}\right)$.

\section{NUMERICAL ANALYSIS OF THE NON-LINEAR EIGENVALUE PROBLEM}

\section{A. The post-gaussian theory without fluctuations}

The coupled non-linear equations (3.16), (3.27) and (3.33) compose an eigenvalue prob-

lem for the eigenvalues $\mu$ and $A_{2}^{(2)}$ which must be solved numerically. The eigenvalues are selected by matching the small- $x$ behaviour, given by (3.36) and (3.37), onto the behaviour at large $x$, equations (2.34) and (3.35). A fourth-order Runge-Kutta integrator is used to integrate (3.27) and (3.33) from near the origin $(x=0.001)$ into the large- $x$, asymptotic regime. Matching onto the proper large- $x$ behaviour for $\mathcal{F}$ is the prime factor determining 
$\mu$; the value of $A_{2}^{(2)}$ controls how well condition (3.35) is satisfied. The techniques used here are very similar to those used in previous studies [3].

We have examined the $O(2)$ model without fluctuations in two and three spatial dimensions. Table \ contains the results for the eigenvalues $\mu$ and $A_{2}^{(2)}$. The upper bound $A_{2}^{(2)}<1 / 2$ is satisfied both here, and later when we include fluctuations. The orderparameter auto-correlation exponent $\lambda$ can be computed using (2.32) once $\mu$ is known. The values for $\lambda$ obtained from the post-gaussian theory are presented in Table $\mathbb{1}$, along with results from the gaussian theory [17]. The gaussian theory is in excellent agreement with simulations [9] of the $O(2)$ model in two dimensions, which give the value $\lambda=1.171$. The post-gaussian theory is in worse agreement with this simulation result. We will see below that the inclusion of fluctuations improves matters slightly.

While the post-gaussian theory decreases $\lambda$ significantly, the form of the order-parameter scaling function $\mathcal{F}$ changes only slightly from the gaussian theory. The function $\mathcal{F}$ is plotted in Fig. 1 for the gaussian and post-gaussian theories in two dimensions. The minor difference between the two theories is reassuring because the gaussian theory is already in good agreement with simulations on this point [1]. The functional form of $f$ has the same qualitative features as $\mathcal{F}$. The quantity $g$ is shown in Fig. 2. A key observation is that the first post-gaussian correction measured by $g(x)$ is small for all $x$.

The function $C_{\psi^{2}}$ (3.18) measuring correlations in the square of the order-parameter field can be calculated from $f$ and $g$. The physical, positive divergence in $C_{\psi^{2}}$ at small- $x$, which is seen in the gaussian theory, is now rendered negative. This occurs in two and three dimensions. One does not have this problem in the post-gaussian scalar theory [3]. Superficially, this unphysical result is a consequence of $A_{2}^{(2)}$ not satisfying the lower bound (3.45). More careful consideration indicates that the root of the problem lies in the method we chose to select $P[\vec{m}]$. Our truncation of the expansion of $P[\vec{m}]$ in Hermite functional polynomials (3.1) ignores terms in $P[\vec{m}]$ that are $\mathcal{O}\left(g^{2}\right)$. To remedy this we use a corrected form for $C_{\psi^{2}}$, 


$$
C_{\psi^{2}}=F_{2}\left(1+g \frac{F_{2}^{\prime}}{F_{2}}\right)^{2}-1
$$

that differs from the previous form (3.18) only at $\mathcal{O}\left(g^{2}\right)$ and is manifestly positive near the origin. The BBS plot of $1 /\left(C_{\psi^{2}}+1\right)$ against $1-\mathcal{F}$ using the corrected form (5.1) is shown in Fig. 3 for the $O(2)$ model in two and three dimensions. The post-gaussian theory is in better agreement with the simulation results [11] than the gaussian theory. The two-dimensional post-gaussian results are in very good agreement with simulation data for $1-\mathcal{F}>0.4$, which are intermediate to large distances, $x>1$. Unlike the gaussian theory, the shape of the BBS plot in the post-gaussian theory depends on spatial dimensionality. On this point the post-gaussian theory exhibits the same trends that are seen in the simulations - as the dimensionality increases from two to three the BBS plot approaches the gaussian result. These observations are strong evidence that post-gaussian statistics provide a more accurate description of the statistics governing the auxiliary field $\vec{m}$ than do gaussian statistics.

Fig. 4 shows the scaling form $\tilde{g}(x)$ (3.50) for the defect-defect correlations for both the post-gaussian and the gaussian theories in two dimensions. Simulation results [15] are also shown. While there still is a divergence in $\tilde{g}(x)$ at small- $x$ in the post-gaussian theory it is weaker than, and of opposite sign to, the divergence occuring in the gaussian theory. The relative weakness of the divergence is a consequence of the small value $K_{2}=0.0018$ in the post-gaussian theory, compared to $K_{2}=-0.5$ in the gaussian theory. We see that the use of post-gaussian statistics does much to eliminate the the unphysical divergence in $\tilde{g}(x)$, even in the absence of fluctuations.

\section{B. The post-gaussian theory including fluctuations}

The purpose of including fluctuations is four-fold. First, we want to render the auxiliary field correlation function analytic for small $x$. Second, we would like to completely eliminate the unphysical divergence at small- $x$ in $\tilde{g}(x)$. Third, we want to improve the agreement between the value of $\lambda$ obtained from the post-gaussian theory and the value seen in sim- 
ulations. Fourth, we would like to see if the inclusion of fluctuations increases $A_{2}^{(2)}$ so that (3.45) is satisfied. We choose $\vec{\Theta}$ to have the form (2.13). In this eigenvalue problem $\omega_{0}$ is selected using (4.7), which guarantees that $K_{2}=L_{2}=0$. We then solve the non-linear eigenvalue problem posed by (3.16), (4.3) and (4.5) using the same methods we used above for the unmodified post-gaussian theory. For $d=2$ the solution of the eigenvalue problem gives $\omega_{0}=-0.2511$, while for $d=3$ one has $\omega_{0}=0.1290$. The eigenvalues $\mu$ and $A_{2}^{(2)}$ are

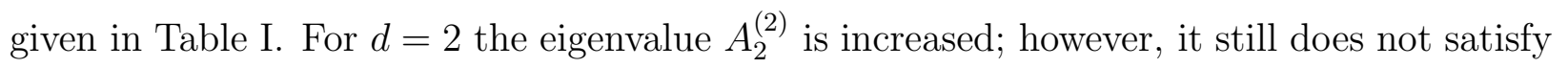
the bound (3.45). In three dimensions $A_{2}^{(2)}$ actually decreases slightly. Table 【1 contains the values for $\lambda$. The agreement between the value for $\lambda$ obtained from the post-gaussian theory for $d=2$ and the value from simulations is slightly improved when fluctuations are added. Adding fluctuations for $d=3$ actually decreases the value for $\lambda$ slightly, moving the post-gaussian result farther away from the gaussian result. We cannot comment on whether or not this represents an improvement since, to our knowledge, no simulation data for $\lambda$ for the three-dimensional $O(2)$ model exists.

The scaling form $\mathcal{F}$ for order-parameter correlations is only slightly modified from the form shown in Fig. 1. On this point, the post-gaussian theory seems less susceptible to the perturbations introduced by the fluctuations than the gaussian theory [17]. Since the bound (3.45) is not satisfied we must again use (5.1) for $C_{\psi^{2}}$ when creating the BBS plot. The results are shown in Fig. 5. It should be noted that fluctuations do not modify the shape of the BBS plot in the gaussian theory because the leading order dependence of $C_{\psi^{2}}$ and $\mathcal{F}$ on $f$ is unaffected by $\vec{u}$, which is $\mathcal{O}\left(L^{-2}\right)$. Again, we see that the post-gaussian theory is in better qualitative agreement with the simulation data than the gaussian theory. However, it appears that the addition of fluctuations removes the dependency of the post-gaussian BBS plot on dimension.

The result for $\tilde{g}(x)$ in the fluctuation-modified theory is shown in Fig. 1 . We see that the inclusion of fluctuations not only eliminates the divergence of $\tilde{g}(x)$ at the origin, but that $\tilde{g}(x)$ is in better agreement with the simulation results down to smaller values of $x$ than it 
was in the unmodified post-gaussian theory. When compared to the gaussian theory with fluctuations [17] we see that the addition of post-gaussian corrections has the desired effect of reducing the magnitude of $\tilde{g}(0)$ and thus producing better agreement with simulation results.

\section{DISCUSSION}

The main achievement of this paper is the extension of the gaussian theory for the $O(2)$ model to allow for non-gaussian statistics. In this sense the theory is unique. It is not trivial that the scaling properties of the gaussian theory are retained. We have demonstrated that when the structural form of $P[\vec{m}]$ is modified to include a post-gaussian part we can still solve the (now double) eigenvalue problem and find the fixed-point solution for $P[\vec{m}]$. Thus we see a structure emerging, related to the nature of the fixed-point, that encourages us to believe that we can systematically change the structural form for $P[\vec{m}]$ via (3.1) and obtain improved results for the scaled quantities. Although the new function $g$ is small, the approach we are attempting here is non-perturbative.

In all areas examined, save the determination of the exponent $\lambda$, the first post-gaussian approximation is in better agreement with the simulation results than the gaussian theory. Scaling results that are already in good agreement with simulations, such as $\mathcal{F}$ and the large scaled-distance behaviour of $\tilde{g}(x)$ are essentially unmodified by the addition of postgaussian corrections. On the other hand in the BBS plot (Fig. 3), where there exists a large discrepancy between simulations [11] and the gaussian theory, the addition of postgaussian terms qualitatively improves matters. Unlike the gaussian theory, the BBS plot in the post-gaussian theory without fluctuations has a dependence on spatial dimensionality which shows the same trends seen in the simulations. In addition, the first post-gaussian approximation nearly removes the divergence in $\tilde{g}(x)$ at small $x$.

The development here suggests some avenues for improving the post-gaussian treatment. For now we have used the ad hoc formula (5.1), which is correct to $\mathcal{O}(g)$, to obtain meaningful 
results for the BBS plot. In order to properly treat these corrections at $\mathcal{O}\left(g^{2}\right)$ we should go to the next order in the post-gaussian approximation sequence. In the current approximation the value for $\lambda$ is decreased from the gaussian result, and one hopes that the systematic inclusion of terms at $\mathcal{O}\left(g^{2}\right)$ will raise $\lambda$ and provide better agreement with simulations. It is apparent that within this approximation scheme the value for $\lambda$ is converging slower than the results for the scaling functions, and may even be experiencing some type of oscillatory behaviour.

It is also a non-trivial matter that we were able to incorporate fluctuations into the postgaussian theory in a consistent manner. We have been able to successfully render both $f$ and $g$ more analytic and thus eliminate the divergence in $\tilde{g}(x)$. The addition of post-gaussian terms to the fluctuation-modified gaussian theory has brought the value for $\tilde{g}(0)$ into better agreement with simulations and thus generally improved the agreement at small-x. The fluctuations also slightly increase the value for $\lambda$. We would also like to go beyond the the simplest post-gaussian fluctuation theory, represented by (2.13). To do this it would seem that one needs to consider $\vec{u}$ as a post-gaussian field, coupled to the post-gaussian $\vec{m}$ [24]. Another interesting effect of adding fluctuations to the post-gaussian theory is the elimination of the dependence of the BBS plot on spatial dimensionality. Whether this is by accident, or is due to some deeper structure in the theory is presently unknown. It would be interesting to see if this effect remains if one includes $\mathcal{O}\left(g^{2}\right)$ terms in $P[\vec{m}]$.

An obvious next step would be to examine the $O(3)$ model using post-gaussian statistics. The $O(3)$ model is interesting to study because of it's application to ferromagnetic materials and the role it plays in the description of monopole defects in nematic liquid crystals and cosmology [25]. There are some interesting new aspects associated with higher $n$ [24].

In examining the $O(2)$ model we have shown how the post-gaussian theory generalizes to a situation with continuous symmetry and solves some of the problems specific to the $n=2$ case. In developing the post-gaussian theory as a systematic and calculable extension of the gaussian theory we have also established the role that the gaussian theory plays as a zerothorder approximation to the true statistics. There are many aspects of this process that 
suggest a deeper underlying structure in the theory, including the interesting interactions between the post-gaussian corrections and the fluctuations.

\section{ACKNOWLEDGMENTS}

R.A.W. gratefully acknowledges support from the NSERC of Canada. This work was supported in part by the MRSEC Program of the National Science Foundation under Award Number DMR-9400379.

\section{APPENDIX:}

In this appendix we outline the method used to evaluate the post-gaussian average occuring in the defect-defect correlation function $g_{d d}(\mathbf{r}, t)(3.49)$. We have

$$
\begin{aligned}
g_{d d}(\mathbf{r}, t) & =\left\langle\delta[\vec{m}(1)] \delta[\vec{m}(2)] \operatorname{det}\left[\vec{\nabla}_{1} \vec{m}(1)\right] \operatorname{det}\left[\vec{\nabla}_{2} \vec{m}(2)\right]\right\rangle \\
& =\epsilon_{i_{1} \cdots i_{n}} \epsilon_{j_{1} \cdots j_{n}}\left\langle D_{i_{1}}(1) \cdots D_{i_{n}}(1) D_{j_{1}}(2) \cdots D_{j_{n}}(2)\right\rangle,
\end{aligned}
$$

with a sum over repeated indices and

$$
D_{i_{q}}(1)=\delta\left[m_{q}(1)\right] \frac{\partial m_{q}(1)}{\partial r_{1 i_{q}}}
$$

Using the definitions (3.5-3.8) and (3.9) the post-gaussian average in (A1) is

$$
\begin{aligned}
& \left\langle D_{i_{1}}(1) \cdots D_{i_{n}}(1) D_{j_{1}}(2) \cdots D_{j_{n}}(2)\right\rangle=\left\langle D_{i_{1}}(1) \cdots D_{i_{n}}(1) D_{j_{1}}(2) \cdots D_{j_{n}}(2)\right\rangle_{0} \\
& +\sum_{k=1}^{n} \int d \overline{1} d \overline{2} A_{2}(\overline{1} \overline{2})\left\langle\frac{\delta^{2}}{\delta m_{k}(\overline{1}) \delta m_{k}(\overline{2})}\left[D_{i_{1}}(1) \cdots D_{i_{n}}(1) D_{j_{1}}(2) \cdots D_{j_{n}}(2)\right]\right\rangle_{0} .
\end{aligned}
$$

The first term on the right-hand side of (A3) is the original gaussian average, and was computed in [14]. We focus on the term involving $A_{2}$, which factors into the following product of $n$ averages over the gaussian distributed scalar $m_{i}$ 's:

$$
\sum_{k=1}^{n} \int d \overline{1} d \overline{2} A_{2}(\overline{1} \overline{2})\left\langle\frac{\delta^{2}}{\delta m_{k}(\overline{1}) \delta m_{k}(\overline{2})}\left[D_{i_{k}}(1) D_{j_{k}}(2)\right]\right\rangle_{0} \prod_{q=1, q \neq k}^{n}\left\langle D_{i_{q}}(1) D_{j_{q}}(2)\right\rangle_{0}
$$


From 14 we know that

$$
\left\langle D_{i_{q}}(1) D_{j_{q}}(2)\right\rangle_{0}=\frac{h}{r} \delta_{i_{q} j_{q}}+\left[\frac{\partial h}{\partial r}-\frac{h}{r}\right] \hat{r}_{i_{q}} \hat{r}_{j_{q}}
$$

with

$$
h=-\frac{\gamma}{2 \pi} \frac{\partial f}{\partial r}
$$

In (A4) the factor involving the integral is evaluated by operating with the functional derivatives, integrating by parts, applying the resulting delta functions and calculating the remaining gaussian integrals. We find

$$
\int d \overline{1} d \overline{2} A_{2}(\overline{1} \overline{2})\left\langle\frac{\delta^{2}}{\delta m_{k}(\overline{1}) \delta m_{k}(\overline{2})}\left[D_{i_{k}}(1) D_{j_{k}}(2)\right]\right\rangle_{0}=\frac{\bar{h}}{r} \delta_{i_{k} j_{k}}+\left[\frac{\partial \bar{h}}{\partial r}-\frac{\bar{h}}{r}\right] \hat{r}_{i_{k}} \hat{r}_{j_{k}} .
$$

with

$$
\bar{h}=-\frac{\gamma}{\pi}\left(\frac{\partial g}{\partial r}+g f \gamma^{2} \frac{\partial f}{\partial r}\right)
$$

Thus the complete expression for $g_{d d}$ is

$$
g_{d d}(\mathbf{r}, t)=n !\left(\frac{h}{r}\right)^{n-1} \frac{\partial h}{\partial r}+\Delta
$$

with the post-gaussian terms contained in

$$
\Delta=\sum_{k=1}^{n} \epsilon_{i_{1} \cdots i_{n}} \epsilon_{j_{1} \cdots j_{n}}\left(\frac{\bar{h}}{r} \delta_{i_{k} j_{k}}+\left[\frac{\partial \bar{h}}{\partial r}-\frac{\bar{h}}{r}\right] \hat{r}_{i_{k}} \hat{r}_{j_{k}}\right) \prod_{q=1, q \neq k}^{n}\left(\frac{h}{r} \delta_{i_{q} j_{q}}+\left[\frac{\partial h}{\partial r}-\frac{h}{r}\right] \hat{r}_{i_{q}} \hat{r}_{j_{q}}\right)
$$

Due to symmetry, the only non-zero contributions to $\Delta$ come from terms containing either zero or exactly two factors of $\hat{r}$. Mindful of this, we evaluate $\Delta$ and obtain

$$
\Delta=n !\left(\frac{h}{r}\right)^{n-2}\left[\frac{\partial \bar{h}}{\partial r} \frac{h}{r}+(n-1) \frac{\bar{h}}{r} \frac{\partial h}{\partial r}\right]
$$

The post-gaussian formula for $g_{d d}$ is then

$$
g_{d d}(\mathbf{r}, t)=n !\left(\frac{h}{r}\right)^{n-1}\left(\frac{\partial h}{\partial r}+\frac{\partial \bar{h}}{\partial r}\right)+(n-1) n !\left(\frac{h}{r}\right)^{n-2} \frac{\bar{h}}{r} \frac{\partial h}{\partial r} .
$$




\section{REFERENCES}

[1] G. F. Mazenko, Phys. Rev. B 42, 4487 (1990).

[2] F. Liu and G. F. Mazenko, Phys. Rev. B 45, 6989 (1992).

[3] G. F. Mazenko, Phys. Rev. E 49, 3717 (1994).

[4] G. F. Mazenko, Phys. Rev. E 50, 3485 (1994).

[5] I. Chuang, B. Yurke, A. N. Pargellis, Phys. Rev. E 47, 3343 (1993); A. N. Pargellis, S. Green, B. Yurke, Phys. Rev. E 49, 4250 (1994).

[6] One example is the q-state clock model treated by F. Liu and G. F. Mazenko, Phys. Rev. B 47, 2866 (1993); C. Sire and S. N. Majumdar, Phys. Rev. E 52, 244 (1995).

[7] There is theoretical and numerical evidence, however, for logarithmic corrections to the growth law when $n=d=2$. It appears that $L \sim(t / \ln t)^{1 / 2}$. B. Yurke, A. N. Pargellis, T. Kovacs, D. A. Huse, Phys. Rev. E 47, 1525 (1993). A. D. Rutenberg and A. J. Bray, Phys. Rev. E 51, 5499 (1995). We see only the $L \sim t^{1 / 2}$ growth law for $n=d=2$ in the theory presented here.

[8] F. Liu and G. F. Mazenko, Phys. Rev. B 44, 9185 (1991).

[9] J.-R. Lee, S. J. Lee, B. Kim, Phys. Rev. E 52, 1550 (1995).

[10] R. E. Blundell, A. J. Bray, S. Sattler Phys. Rev. E 48, 2476 (1993).

[11] R. E. Blundell and A. J. Bray, Phys. Rev. E 49, 4925 (1994).

[12] This is not because one cannot determine the pre-factor theoretically but because of the difficulty of discrete lattices used in simulations.

[13] In addition to the approach taken here, work towards a systematic treatment of phaseordering kinetics has been undertaken in A. J. Bray and K. Humayun, Phys. Rev. E 48, R1609 (1993). 
[14] F. Liu and G. F. Mazenko, Phys. Rev. B 46, 5963 (1992).

[15] M. Mondello and N. Goldenfeld, Phys. Rev. A 42, 5865 (1990).

[16] T. Nagaya, H. Orihara, Y. Ishibashi, J. Phys. Soc. Jpn. 64, 78 (1995).

[17] G. F. Mazenko and R. A. Wickham (submitted to Phys. Rev. E).

[18] Scaling in the $O(n)$ model is believed to be controlled by a zero-temperature fixed point. Near the fixed point, temperature is an irrelevant variable and is not expected to affect the scaling exponents or the leading order form for the scaling functions. A. J. Bray, Phys. Rev. Lett. 62, 2841 (1989); Phys. Rev. B 41, 6724 (1990).

[19] Typically, $V$ is taken to have the form: $V=\frac{1}{4}\left(\psi_{0}^{2}-\psi^{2}\right)^{2}$.

[20] There is a mass term in the equation for $\vec{u}_{T}$, but it is $\mathcal{O}\left(L^{-2}\right)$.

[21] For a treatment that includes spin-waves excited by a non-zero temperature see E. M. Kramer, Phys. Rev. E 50, 3594 (1994).

[22] A. J. Bray and S. Puri, Phys. Rev. Lett. 67, 2670 (1991); H. Toyoki, Phys. Rev. B 45, 1965 (1992).

[23] This relation between $L^{2}(t)$ and $S_{0}(t)$, which introduces the constant $\mu$ is the same relation as in [2]. In other work [1] one defines $\mu$ through the relations $L \dot{L}=\mu, L^{2}=\pi S_{0}$. This approach is physically appealing because $\mu$ then determines the pre-factor to the power-law growth: $L=\sqrt{2 \mu} t^{1 / 2}$. Either approach, however, is valid.

[24] R. A. Wickham and G. F. Mazenko (unpublished).

[25] A. Pargellis, N. Turok, B. Yurke, Phys. Rev. Lett. 67, 1570 (1991). 


\section{TABLES}

TABLE I. Values for the eigenvuales $\mu$ (top section) and $A_{2}^{(2)}$ (bottom section) for the post-gaussian theory, both with and without fluctuations.

\begin{tabular}{lll}
\hline \hline & $\omega_{0}=0$ & $\omega_{0} \neq 0$ \\
\hline$d=2$ & 0.8380 & 0.7597 \\
$d=3$ & 0.5326 & 0.5525 \\
\hline$d=2$ & 0.05861 & 0.08092 \\
$d=3$ & 0.06364 & 0.05392 \\
\hline \hline
\end{tabular}

TABLE II. Auto-correlation exponent $\lambda$ from the gaussian theory [17] (top section) and from the post-gaussian theory (bottom section), both with and without fluctuations.

\begin{tabular}{lcc}
\hline \hline & $\omega_{0}=0$ & $\omega_{0} \neq 0$ \\
\hline$d=2$ & 1.172 & 1.269 \\
$d=3$ & 1.618 & 1.655 \\
\hline$d=2$ & 1.063 & 1.092 \\
$d=3$ & 1.525 & 1.513 \\
\hline \hline
\end{tabular}




\section{FIGURES}

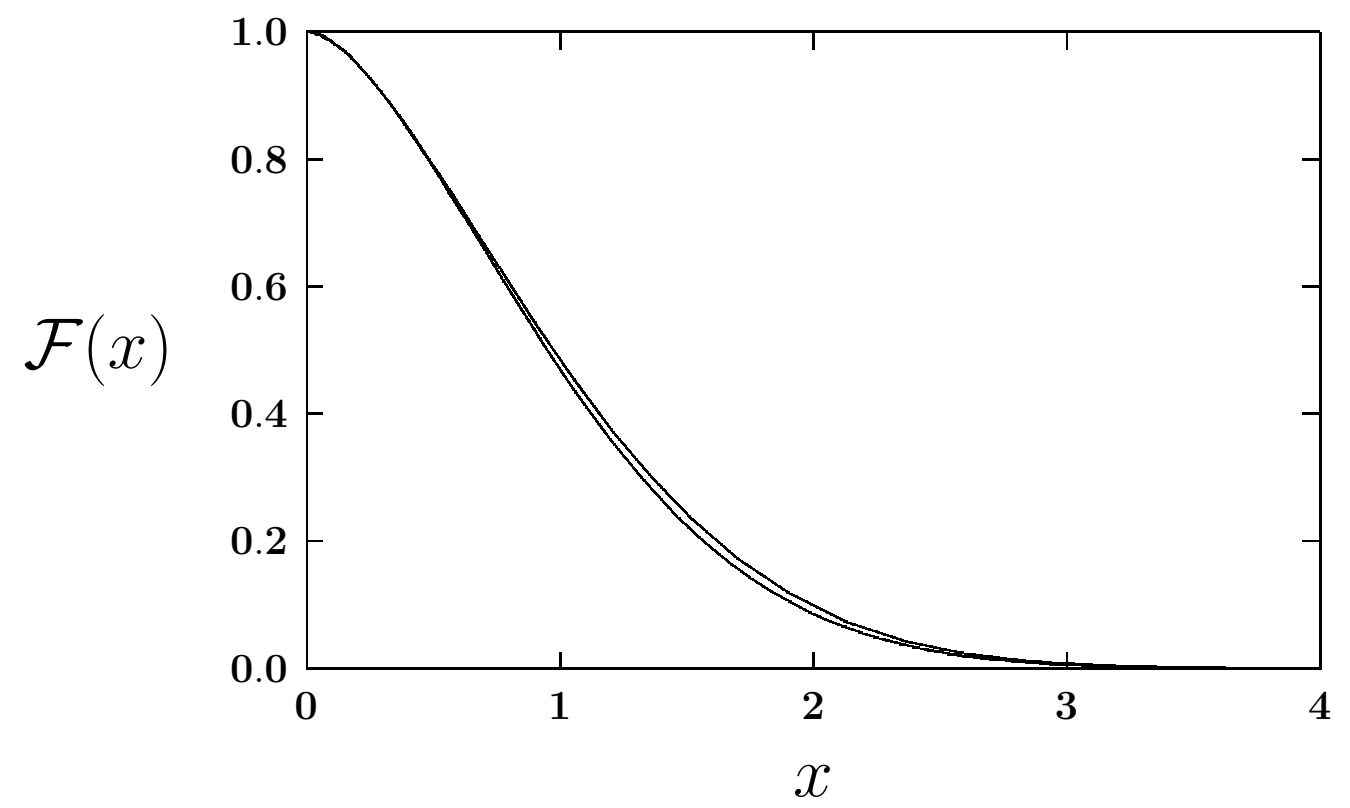

FIG. 1. Scaling form $\mathcal{F}(x)$ for the order-parameter correlation function in two dimensions. At $x=2$ the upper curve is the post-gaussian theory without fluctuations and the lower curve is the gaussian theory without fluctuations. 


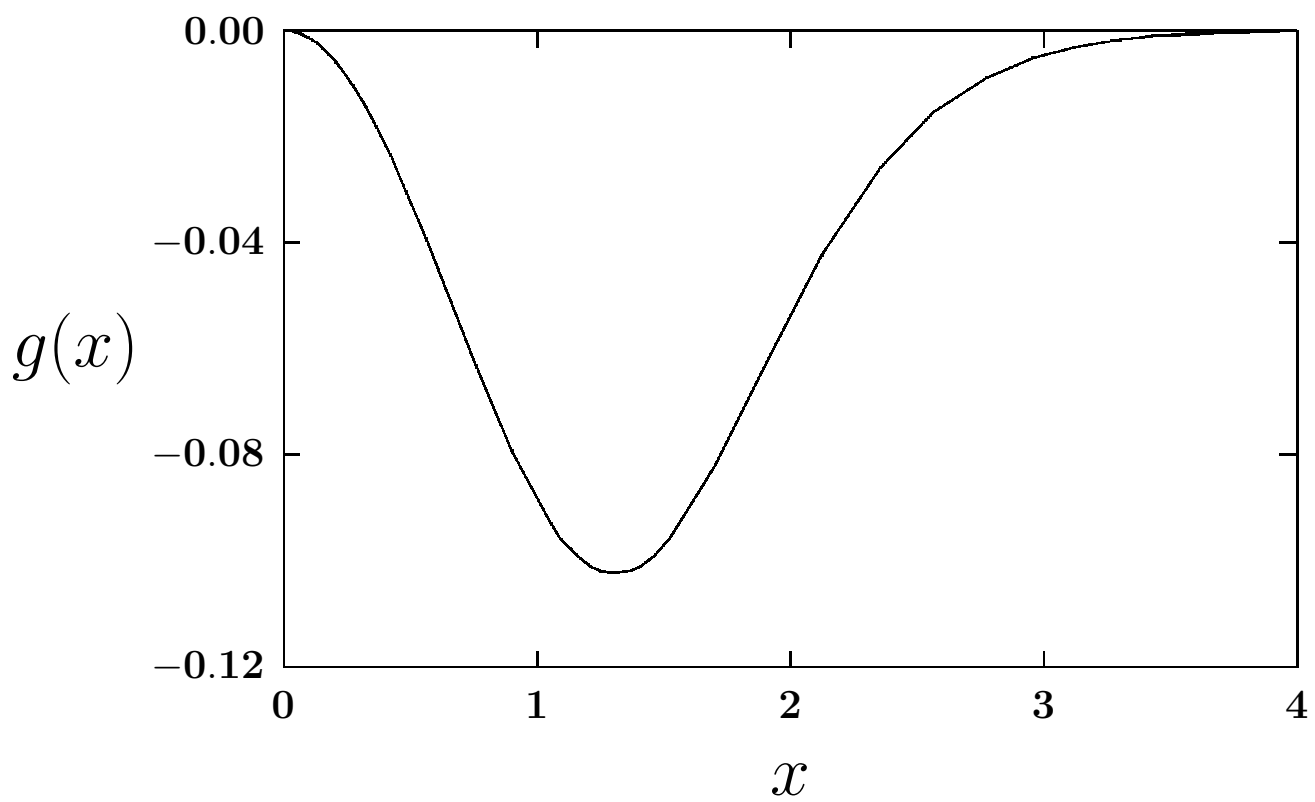

FIG. 2. The auxiliary function $g(x)$ for the post-gaussian theory without fluctuations in two dimensions. 


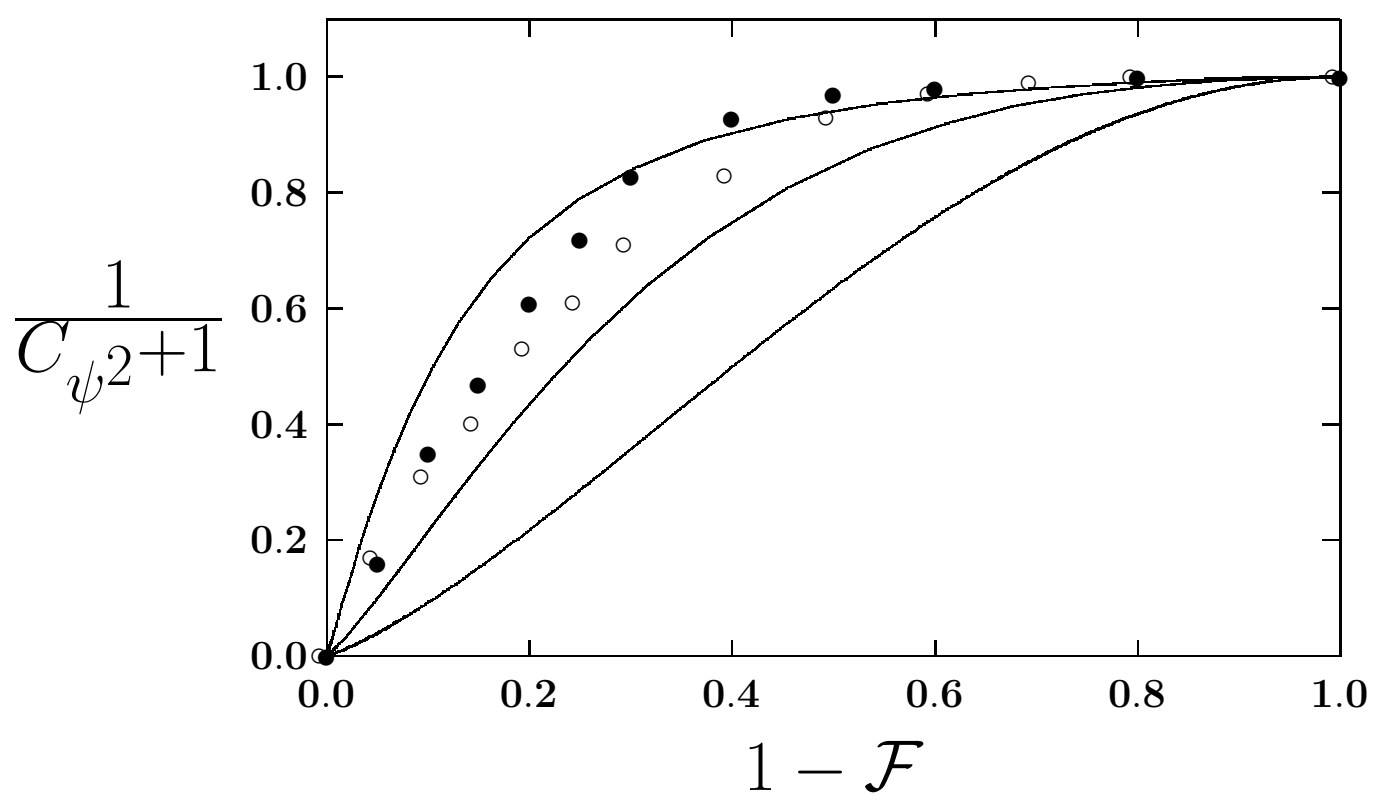

FIG. 3. BBS plot for the gaussian and post-gaussian theories without fluctuations. At $1-\mathcal{F}=0.2$ the upper solid curve is the post-gaussian result for $d=2$, the middle curve is for $d=3$ and the lower curve is the gaussian result. The solid circles are the simulation data for $d=2$ and the open circles are the simulation data for $d=3[11$. 


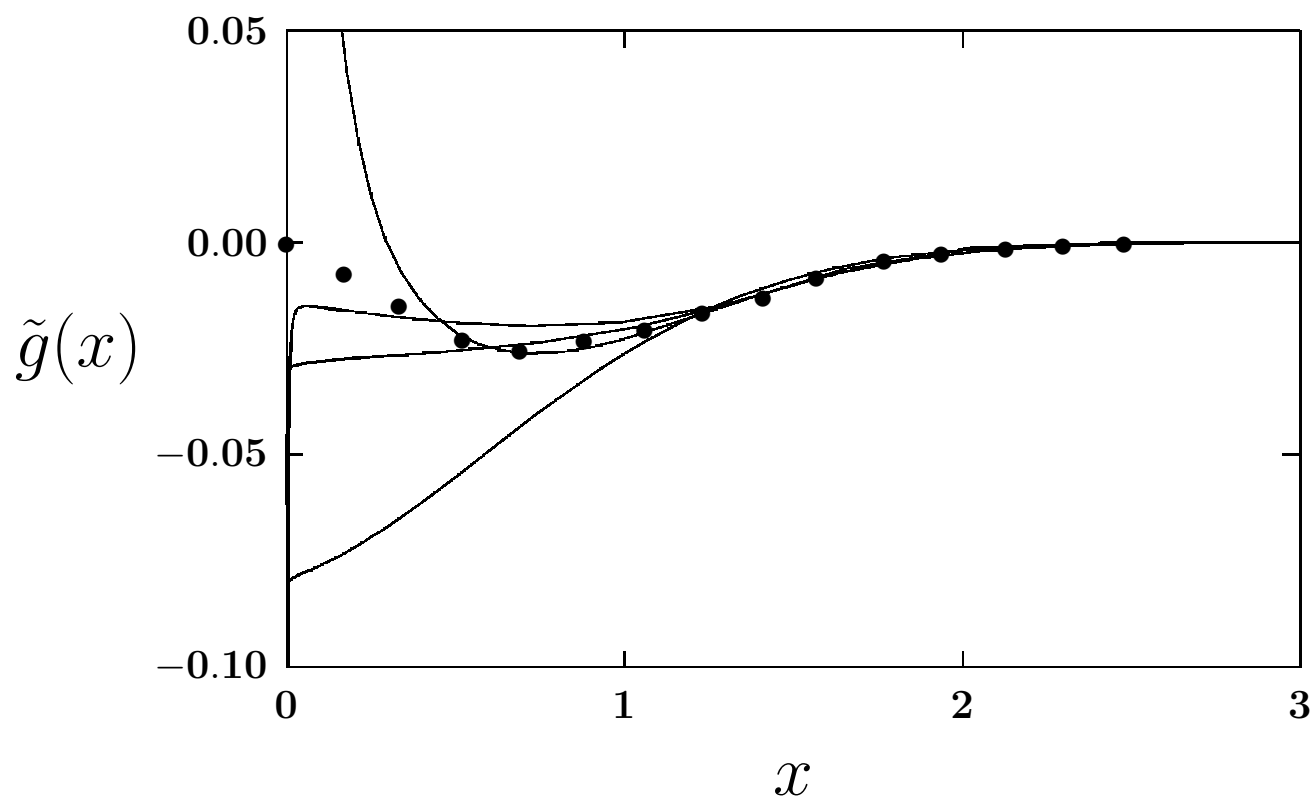

FIG. 4. Scaling function $\tilde{g}(x)$ for the defect-defect correlations in two dimensions. At $x=0.1$, from bottom to top, the solid curves represent: the gaussian theory with $\omega_{0} \neq 0$ [17]; the post-gaussian theory with $\omega_{0} \neq 0$; the post-gaussian theory without fluctuations (diverging negatively); the gaussian theory without fluctuations (diverging positively) [14]. The dots represent the simulation data 15 . 


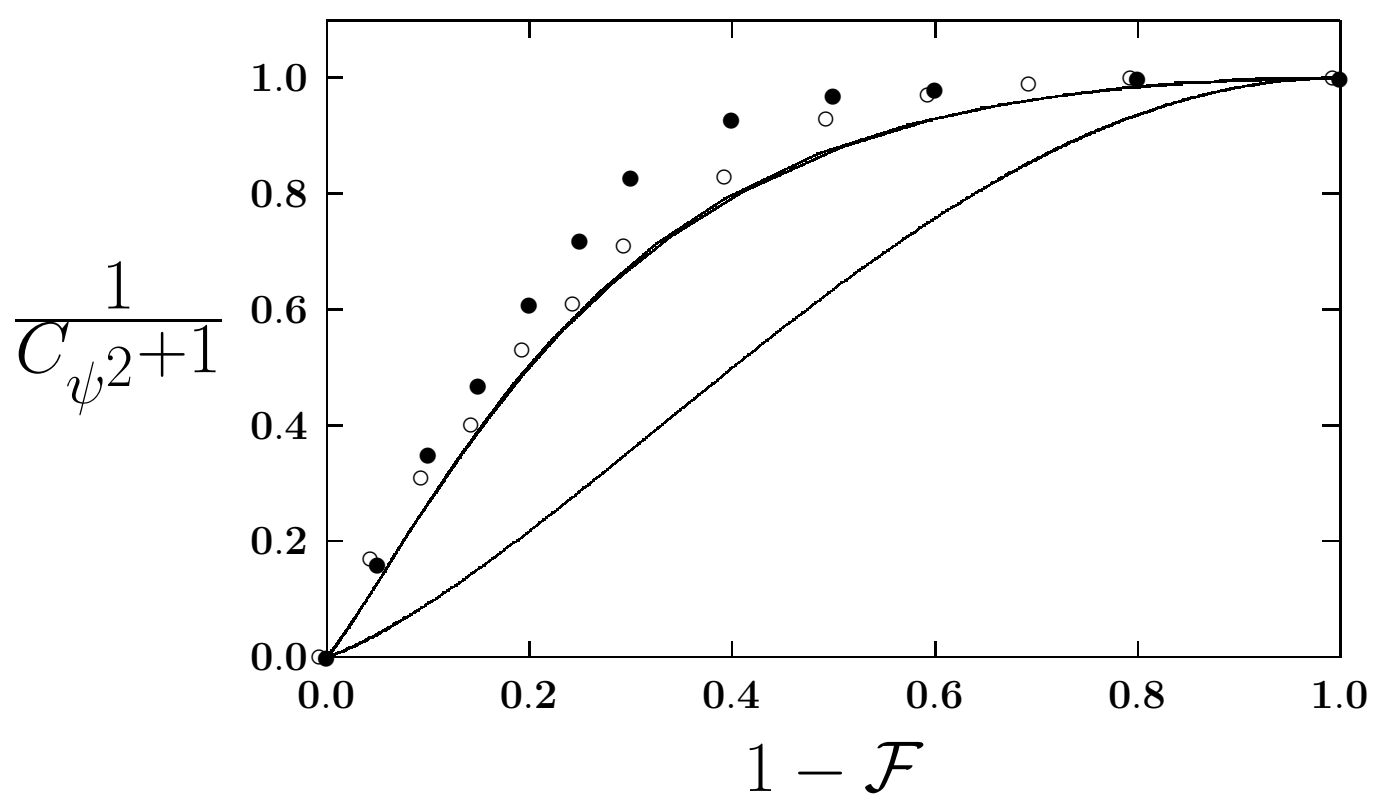

FIG. 5. BBS plot for the post-gaussian theory including fluctuations. At $1-\mathcal{F}=0.5$ the lower solid curve is the gaussian theory and the upper two curves, which are virtually indistinguishable, are the post-gaussian theory with $\omega_{0} \neq 0$ for $d=2$ and $d=3$. The solid circles are the simulation data for $d=2$ and the open circles are the simulation data for $d=3$ [11. 